\title{
Existence and Global Exponential Stability of Pseudo Almost Periodic Solutions for Neutral Type Quaternion-Valued Neural Networks with Delays in the Leakage Term on Time Scales
}

\author{
Yongkun Li and Xiaofang Meng \\ Department of Mathematics, Yunnan University, Kunming, Yunnan 650091, China \\ Correspondence should be addressed to Yongkun Li; yklie@ynu.edu.cn
}

Received 10 September 2017; Accepted 22 November 2017; Published 26 December 2017

Academic Editor: Sigurdur F. Hafstein

Copyright ( 12017 Yongkun Li and Xiaofang Meng. This is an open access article distributed under the Creative Commons Attribution License, which permits unrestricted use, distribution, and reproduction in any medium, provided the original work is properly cited.

\begin{abstract}
We propose a class of neutral type quaternion-valued neural networks with delays in the leakage term on time scales that can unify the discrete-time and the continuous-time neural networks. In order to avoid the difficulty brought by the noncommutativity of quaternion multiplication, we first decompose the quaternion-valued system into four real-valued systems. Then, by applying the exponential dichotomic theory of linear dynamic equations on time scales, Banach's fixed point theorem, the theory of calculus on time scales, and inequality techniques, we obtain some sufficient conditions on the existence and global exponential stability of pseudo almost periodic solutions for this class of neural networks. Our results are completely new even for both the case of the neural networks governed by differential equations and the case of the neural networks governed by difference equations and show that, under a simple condition, the continuous-time quaternion-valued network and its corresponding discrete-time quaternionvalued network have the same dynamical behavior for the pseudo almost periodicity. Finally, a numerical example is given to illustrate the feasibility of our results.
\end{abstract}

\section{Introduction}

The quaternion, which was discovered by the Irish mathematician Hamilton [1] in order to generalize complex number properties to multidimensional space, is extensively used in several fields, such as modern mathematics, physics, and computer graphics [2-4]. One of the advantages by the use of quaternions is that it can treat and operate three- or fourdimensional vectors as one entity, which allows a significant decrease of computational complexity in three- or fourdimensional problems, so the effective information processing can be achieved by the operations for quaternionic variables. Therefore, the quaternion-valued neural network is able to cope with multidimensional issues more efficiently by employing quaternion directly.

In this respect, the quaternion-valued neural network is a fast growing field of research in both theoretical and application points of view (see [5-9]). Quaternion neural networks have been widely used in many fields and demonstrated better performances than the real number neural networks in chaotic time series prediction [10], approximate quaternionvalued functions [11], 3D wind forecasting [12, 13], image processing $[14,15]$, color-face recognition [16], vector sensor processing [17], and so on.

In reality, it is well known that the time delay is inevitable. In the circuit implementation of neural networks, time delays occur naturally due to the processing and transmission of signals in the network and the finite switching speed of amplifiers. And they may change the dynamical behaviors of considered neural networks. Therefore, the consideration of time delays is more and more significant in the study of the dynamics of neural networks.

Many scholars have devoted themselves into the dynamics analysis of neural networks with various types of time delays and many valuable results have been achieved in the existing literature see [18-26]. There are three typical types of time delays for incorporating time delays into neural networks: (i) introduce transmission delays into the neural 
networks, and consider discrete delays, distributed delays, mixed delays, even state depended delays, or complex delays; (ii) consider the delays in the leakage term; (iii) take into account neutral type delays. All of the above three types of time delays may alter the dynamics of the neural network under consideration.

On the one hand, the concept of pseudo-almost periodicity was introduced by Zhang $[27,28]$ in the early 1990 s. It quickly aroused the interest of some mathematical researchers [29-31]. The pseudo almost periodicity is more general and complicated than the periodicity and the almost periodicity. In the last few years, the pseudo almost periodicity has become a hot research topic, especially for the pseudo almost periodic oscillation of neural networks [32-39].

On the other hand, as it is known, both continuous-time and discrete-time neural networks are important in theocratic studies and applications. Moreover, discrete-time neural networks are more convenient for computation and numerical simulation than continuous-time neural networks. Therefore, we must not only study continuous-time neural networks, but also study discrete-time neural networks. Fortunately, the theory of time scales, which was initiated by Hilger [40] in his Ph.D. thesis in 1988, can unify the continuous and discrete cases. Studying dynamic equations on time scales can unify the differential equation case and the difference equation case. In recent years, the time scale theory has been widely concerned and rapidly developed [41-45]. And, many authors have studied the dynamical behavior of neural networks on time scales [46-54].

However, to the best of our knowledge, there is no paper published on the existence and stability of pseudo almost periodic solutions of quaternion-valued neural networks on time scales. This is important in theory and application, and it is also a very challenging issue.

Motivated by the above statement, in this paper, we propose the following neutral type quaternion-valued neural network with delays in the leakage term on time scales:

$$
\begin{aligned}
x_{p}^{\Delta}(t)= & -c_{p}(t) x_{p}\left(t-\delta_{p}(t)\right) \\
& +\sum_{q=1}^{n} a_{p q}(t) f_{q}\left(x_{q}\left(t-\tau_{p q}(t)\right)\right) \\
& +\sum_{q=1}^{n} b_{p q}(t) g_{q}\left(x_{q}^{\Delta}\left(t-\eta_{p q}(t)\right)\right)+u_{p}(t),
\end{aligned}
$$

$$
t \in \mathbb{T},
$$

where $\mathbb{T}$ is an almost periodic time scale, $p \in\{1,2, \ldots, n\}:=$ $\Lambda, x_{p}(t) \in \mathbb{H} \otimes \mathbb{T}$ is the state of the $p$ th neuron at time $t ; c_{p}(t)>$ 0 is the self-feedback connection weight, $\mathbb{M} \otimes \mathbb{T}$ denotes the set of all quaternion-valued functions defined on time scale $\mathbb{T}$; $a_{p q}(t)$ and $b_{p q}(t) \in \mathbb{U} \otimes \mathbb{T}$ are the delay connection weight and the neutral delay connection weight from neuron $q$ to neuron $p$ at time $t$, respectively; $u_{p}(t)$ is an external input on the $p$ th unit at time $t ; \delta_{p}(t)$ denotes the leakage delay satisfying $t-$ $\delta_{p}(t) \in \mathbb{T}$ for $t \in \mathbb{T} ; \tau_{p q}(t)$ and $\eta_{p q}(t)$ are transmission delays satisfying $t-\tau_{p q}(t) \in \mathbb{T}$ and $t-\eta_{p q}(t) \in \mathbb{T}$ for $t \in \mathbb{T}$.
The initial condition of system (1) is of the form

$$
x_{p}(s)=\varphi_{p}(s), \quad p \in \Lambda, s \in[-\theta, 0]_{\mathbb{T}},
$$

where $\theta=\max \{\delta, \tau, \eta\}, \delta=\max _{p \in \Lambda}\left\{\sup _{t \in \mathbb{T}} \delta_{p}(t)\right\}, \tau=$ $\max _{p, q \in \Lambda}\left\{\sup _{t \in \mathbb{N}} \tau_{p q}(t)\right\}, \eta=\max _{p, q \in \Lambda}\left\{\sup _{t \in \mathbb{T}} \eta_{p q}(t)\right\}, \varphi_{p}(s) \epsilon$ $C\left([-\theta, 0]_{\mathbb{T}}, \mathbb{\mathbb { T } ^ { n }} \otimes \mathbb{T}\right)$.

Throughout this paper, we denote $[a, b]_{\mathbb{T}}=\{t \mid t \in[a, b] \cap$ $\mathbb{T}\}$. For convenience, for an rd-continuous pseudo almost periodic function $f: \mathbb{T} \rightarrow \mathbb{R}$, we denote $f^{-}=\inf _{t \in \mathbb{T}}|f(t)|$ and $f^{+}=\sup _{t \in \mathbb{T}}|f(t)|$.

Our main purpose of this paper is to study the existence and global exponential stability of pseudo almost periodic solutions of (1). Our results are completely new even for both the case of the neural networks governed by quaternionvalued differential equations and the case of the neural networks governed by quaternion-valued difference equations.

The rest of this paper is organized as follows. In Section 2, we introduce some definitions and preliminary lemmas and transform the quaternion-valued system (1) into four realvalued systems. In Section 3, we establish some sufficient conditions for the existence and global exponential stability of pseudo almost periodic solutions of (1). In Section 4, we give an example to demonstrate the feasibility of our results. This paper ends with a brief conclusion in Section 5.

\section{Preliminaries}

In this section, we shall first recall some fundamental definitions, lemmas which are used in what follows.

The skew field of quaternions is denoted by

$$
\mathbb{H}:=\left\{q=q^{R}+q^{I} i+q^{J} j+q^{K} k\right\},
$$

where $q^{R}, q^{I}, q^{J}$, and $q^{K}$ are real numbers and the elements $i$, $j$, and $k$ obey Hamilton's multiplication rules:

$$
\begin{aligned}
& i j=-j i=k, \\
& j k=-k j=i, \\
& k i=-i k=j, \\
& i^{2}=j^{2}=k^{2}=i j k=-1 .
\end{aligned}
$$

The quaternion conjugate is defined by $\bar{q}=q^{R}-q=q^{R}-q^{I} i-$ $q^{J} j-q^{K} k$, and the norm $|q|$ of $q$ is defined as $|q|^{2}=q \bar{q}=\bar{q} q=$ $\left(q^{R}\right)^{2}+\left(q^{I}\right)^{2}+\left(q^{J}\right)^{2}+\left(q^{K}\right)^{2}$.

A time scale $\mathbb{T}$ is an arbitrary nonempty closed subset of the real set $\mathbb{R}$ with the topology and ordering inherited from $\mathbb{R}$. The forward jump operator $\sigma: \mathbb{T} \rightarrow \mathbb{T}$ is defined by $\sigma(t)=$ $\inf \{s \in \mathbb{T}, s>t\}, t \in \mathbb{T}$, while the backward jump operator $\rho: \mathbb{T} \rightarrow \mathbb{T}$ is defined by $\rho(t)=\sup \{s \in \mathbb{T}, s<t\}, t \in \mathbb{T}$, and the graininess function $\mu: \mathbb{T} \rightarrow[0, \infty)$ is defined by $\mu(t)=\sigma(t)-t$.

The point $t \in \mathbb{T}$ is called left-dense, left-scattered, rightdense, or right-scattered if $\rho(t)=t, \rho(t)<t, \sigma(t)=t$, or $\sigma(t)>t$, respectively. Points that are right-dense and left-dense at the same time are called dense. If $\mathbb{T}$ has a leftscattered maximum $m$, define $\mathbb{T}^{\kappa}=\mathbb{T}-\{m\}$; otherwise, 
set $\mathbb{T}^{\kappa}=\mathbb{T}$. If $\mathbb{T}$ has a right-scattered maximum $m$, define $\mathbb{T}_{\kappa}=\mathbb{T}-\{m\}$; otherwise, set $\mathbb{T}_{\kappa}=\mathbb{T}$.

Assume that $f: \mathbb{T} \rightarrow \mathbb{R}$ is a function and let $t \in \mathbb{T}^{k}$. Then we define $f^{\Delta}(t)$ to be the number (provided its exists) with the property that, given any $\varepsilon>0$, there is a neighborhood $U$ of $t$ such that

$$
\left|f(\sigma(t))-f(s)-f^{\Delta}(t)(\sigma(t)-s)\right| \leq \varepsilon|\sigma(t)-s|,
$$

for all $s \in U$. We call $f^{\Delta}(t)$ the delta derivative of $f$ at $t$. Moreover, we say that $f$ is delta differentiable on $\mathbb{T}^{k}$ provided that $f^{\Delta}(t)$ exists for all $t \in \mathbb{T}^{k}$.

By writing $f \in \mathbb{U} \otimes \mathbb{\Psi}$ in the form of $f=f^{R}+i f^{I}+j f^{I}+k f^{K}$ with $f^{l} \in \mathbb{U} \otimes \mathbb{T}, l \in\{R, I, J, K\}:=E$, it is easy to verify that $f$ is delta differentiable if and only if $f^{R}, f^{I}, f^{J}, f^{K}$ are delta differentiable. Moreover, if $f$ is delta differentiable, then

$$
\begin{aligned}
f^{\Delta}(t)= & \left(f^{R}\right)^{\Delta}(t)+i\left(f^{I}\right)^{\Delta}(t)+j\left(f^{J}\right)^{\Delta}(t) \\
& +k\left(f^{K}\right)^{\Delta}(t) .
\end{aligned}
$$

A function $p: \mathbb{T} \rightarrow \mathbb{R}$ is said to be regressive provided $1+\mu(t) p(t) \neq 0$ for all $t \in \mathbb{T}^{\kappa}$. The set of all regressive and rdcontinuous functions $p: \mathbb{T} \rightarrow \mathbb{R}$ is denoted by $\mathscr{R}=\mathscr{R}(\mathbb{T})$. We define $\mathscr{R}^{+}=\{p \in \mathscr{R}: 1+\mu(t) p(t)>0$ for all $t \in \mathbb{T}\}$. For more knowledge about calculus on time scales, we refer to $[41,42]$.

Definition 1 (see [47]). A time scale $\mathbb{T}$ is called an almost periodic time scale if

$$
\Pi:=\{\tau \in \mathbb{R}: t \pm \tau \in \mathbb{T}, \forall t \in \mathbb{T}\} \neq\{0\} .
$$

Definition 2 (see [47]). Let $\mathbb{T}$ be an almost periodic time scale. A function $f \in C\left(\mathbb{T}, \mathbb{R}^{n}\right)$ is called an almost periodic on $\mathbb{T}$ if for any given $\varepsilon>0$, there exists a constant $l(\varepsilon)>0$ such that each interval of length $l(\varepsilon)$ contains at least one $\tau(\varepsilon) \in \Pi$ such that

$$
|f(t+\tau)-f(t)|<\varepsilon, \quad \forall t \in \mathbb{T} .
$$

Let $\mathrm{AP}\left(\mathbb{T}, \mathbb{R}^{n}\right)=\left\{f \in C\left(\mathbb{T}, \mathbb{R}^{n}\right): f\right.$ be almost periodic $\}$ and $\mathrm{BC}\left(\mathbb{T}, \mathbb{R}^{n}\right)$ denote the space of all bounded continuous functions from $\mathbb{T}$ to $\mathbb{R}^{n}$.

Similar to Definition 4.1 in [55], we introduce the following definition.

Definition 3. A function $f \in C\left(\mathbb{T}, \mathbb{R}^{n}\right)$ is called pseudo almost periodic if $f=g+h$, where $g \in \operatorname{AP}\left(\mathbb{T}, \mathbb{R}^{n}\right)$ and $h \in \operatorname{PAP}_{0}\left(\mathbb{T}, \mathbb{R}^{n}\right)=\left\{f \in \mathrm{BC}\left(\mathbb{T}, \mathbb{R}^{n}\right): f\right.$ is $\Delta$-measurable such that $\lim _{r \rightarrow+\infty}(1 / 2 r) \int_{t_{0}-r}^{t_{0}+r}|f(s)| \Delta s=0$, where $\left.t_{0} \in \mathbb{T}, r \in \Pi\right\}$.

We denote by $\operatorname{PAP}\left(\mathbb{T}, \mathbb{R}^{n}\right)$ the set of all pseudo almost periodic functions defined on $\mathbb{T}$.

Lemma 4 (see [56]). If $f, g \in P A P\left(\mathbb{T}, \mathbb{R}^{n}\right)$, then $f+g, f g \in$ $P A P\left(\mathbb{T}, \mathbb{R}^{n}\right)$; if $f \in P A P\left(\mathbb{T}, \mathbb{R}^{n}\right), g \in A P\left(\mathbb{T}, \mathbb{R}^{n}\right)$, then $f g \in$ $\operatorname{PAP}\left(\mathbb{T}, \mathbb{R}^{n}\right)$.
Similar to the proof of Lemma 2.10 in [56], one can show the following.

Lemma 5. If $f \in C(\mathbb{R}, \mathbb{R})$ satisfies the Lipschitz condition, $\varphi \in$ $P A P(\mathbb{T}, \mathbb{R}), \tau \in C^{1}(\mathbb{T}, \Pi)$, and $\inf _{t \in \mathbb{T}}\left(1-\tau^{\Delta}(t)\right)>0$, then $f(\varphi(t-\tau(t))) \in \operatorname{PAP}(\mathbb{\mathbb { T }}, \mathbb{R})$.

Definition 6. Function $f=f^{R}+i f^{I}+j f^{J}+k f^{K} \in C\left(\mathbb{T}\right.$, $\mathbb{H}^{n} \otimes$ $\mathbb{T})$ is called pseudo almost periodic if for each $l \in E, f^{l} \in$ $C\left(\mathbb{T}, \mathbb{R}^{n}\right)$ is pseudo almost periodic.

Definition 7 (see [47]). Let $A(t)$ be an $n \times n$ matrix-valued function on $\mathbb{T}$. Then the linear system

$$
x^{\Delta}(t)=A(t) x(t), \quad t \in \mathbb{T},
$$

is said to admit an exponential dichotomy on $\mathbb{T}$ if there exist positive constant $K, \alpha$, projection $P$, and the fundamental solution matrix $X(t)$ of (9), satisfying

$$
\left\|X(t) P X^{-1}(s)\right\|_{0} \leq K e_{\ominus \alpha}(t, s),
$$

$s, t \in \mathbb{T}, t \geq s$,

$$
\left\|X(t)(I-P) X^{-1}(s)\right\|_{0} \leq K e_{\ominus \alpha}(s, t),
$$

$s, t \in \mathbb{T}, t \leq s$.

Consider the following pseudo almost periodic system:

$$
x^{\Delta}(t)=A(t) x(t)+f(t), \quad t \in \mathbb{T},
$$

where $A(t)$ is an almost periodic matrix function and $f(t)$ is a pseudo almost periodic vector function.

Lemma 8 (see [47]). If the linear system (9) admits an exponential dichotomy, then the pseudo almost periodic system (11) has a unique pseudo almost periodic solution $x(t)$ as follows:

$$
\begin{aligned}
x(t)= & \int_{-\infty}^{t} X(t) P X^{-1}(\sigma(s)) f(s) \Delta s \\
& -\int_{t}^{+\infty} X(t)(I-P) X^{-1}(\sigma(s)) f(s) \Delta s,
\end{aligned}
$$

where $X(t)$ is the fundamental solution matrix of (9).

Lemma 9 (see [46]). Let $c_{p}(t): \mathbb{T} \rightarrow \mathbb{R}^{+}$be an almost periodic function, $-c_{p}(t) \in \mathscr{R}^{+}, p \in \Lambda, t \in \mathbb{T}$, and $\min _{1 \leq p \leq n}\left\{\inf _{t \in \mathbb{T}} c_{p}(t)\right\}>0$; then the linear system

$$
x^{\Delta}(t)=\operatorname{diag}\left(-c_{1}(t),-c_{2}(t), \ldots,-c_{n}(t)\right) x(t)
$$

admits an exponential dichotomy on $\mathbb{T}$. 

ing:

Throughout the rest of this paper, we assume the follow-

$\left(H_{1}\right)$ Let $x_{p}=x_{p}^{R}+i x_{p}^{I}+j x_{p}^{J}+k x_{p}^{K}$, where $x_{p}^{R}, x_{p}^{I}, x_{p}^{J}, x_{p}^{K} \in$ $\mathbb{R}, p \in \Lambda$. Then $f_{p}\left(x_{p}\right)$ and $g_{p}\left(x_{p}\right)$ can be expressed as

$$
\begin{aligned}
f_{p}\left(x_{p}\right)= & f_{p}^{R}\left(x_{p}^{R}\right)+i f_{p}^{I}\left(x_{p}^{I}\right)+j f_{p}^{J}\left(x_{p}^{J}\right) \\
& +k f_{p}^{K}\left(x_{p}^{K}\right), \quad p \in \Lambda, \\
g_{p}\left(x_{p}\right)= & g_{p}^{R}\left(x_{p}^{R}\right)+i g_{p}^{I}\left(x_{p}^{I}\right)+j g_{p}^{J}\left(x_{p}^{J}\right) \\
& +k g_{p}^{K}\left(x_{p}^{K}\right), \quad p \in \Lambda .
\end{aligned}
$$

By $\left(H_{1}\right)$, we can transform system (1) into the following four real-valued systems:

$$
\begin{aligned}
& \left(x_{p}^{R}(t)\right)^{\Delta}=-c_{p}(t) x_{p}^{R}\left(t-\delta_{p}(t)\right) \\
& +\sum_{q=1}^{n}\left(a_{p q}^{R}(t) f_{q}^{R}\left(x_{q}^{R}\left(t-\tau_{p q}(t)\right)\right)\right. \\
& -a_{p q}^{I}(t) f_{q}^{I}\left(x_{q}^{I}\left(t-\tau_{p q}(t)\right)\right) \\
& -a_{p q}^{J}(t) f_{q}^{J}\left(x_{q}^{J}\left(t-\tau_{p q}(t)\right)\right) \\
& \left.-a_{p q}^{K}(t) f_{q}^{K}\left(x_{q}^{K}\left(t-\tau_{p q}(t)\right)\right)\right) \\
& +\sum_{q=1}^{n}\left(b_{p q}^{R}(t) g_{q}^{R}\left(\left(x_{q}^{R}\right)^{\Delta}\left(t-\eta_{p q}(t)\right)\right)\right. \\
& -b_{p q}^{I}(t) g_{q}^{I}\left(\left(x_{q}^{I}\right)^{\Delta}\left(t-\eta_{p q}(t)\right)\right) \\
& -b_{p q}^{J}(t) g_{q}^{J}\left(\left(x_{q}^{J}\right)^{\Delta}\left(t-\eta_{p q}(t)\right)\right) \\
& \left.-b_{p q}^{K}(t) g_{q}^{K}\left(\left(x_{q}^{K}\right)^{\Delta}\left(t-\eta_{p q}(t)\right)\right)\right)+u_{p}^{R}(t) \text {, } \\
& \left(x_{p}^{I}(t)\right)^{\Delta}=-c_{p}(t) x_{p}^{I}\left(t-\delta_{p}(t)\right) \\
& +\sum_{q=1}^{n}\left(a_{p q}^{R}(t) f_{q}^{I}\left(x_{q}^{I}\left(t-\tau_{p q}(t)\right)\right)\right. \\
& +a_{p q}^{I}(t) f_{q}^{R}\left(x_{q}^{R}\left(t-\tau_{p q}(t)\right)\right) \\
& +a_{p q}^{J}(t) f_{q}^{K}\left(x_{q}^{K}\left(t-\tau_{p q}(t)\right)\right) \\
& \left.-a_{p q}^{K}(t) f_{q}^{J}\left(x_{q}^{J}\left(t-\tau_{p q}(t)\right)\right)\right) \\
& +\sum_{q=1}^{n}\left(b_{p q}^{R}(t) g_{q}^{I}\left(\left(x_{q}^{I}\right)^{\Delta}\left(t-\eta_{p q}(t)\right)\right)\right. \\
& +b_{p q}^{I}(t) g_{q}^{R}\left(\left(x_{q}^{R}\right)^{\Delta}\left(t-\eta_{p q}(t)\right)\right)
\end{aligned}
$$

$$
\begin{aligned}
& +b_{p q}^{J}(t) g_{q}^{K}\left(\left(x_{q}^{K}\right)^{\Delta}\left(t-\eta_{p q}(t)\right)\right) \\
& \left.-b_{p q}^{K}(t) g_{q}^{J}\left(\left(x_{q}^{J}\right)^{\Delta}\left(t-\eta_{p q}(t)\right)\right)\right)+u_{p}^{I}(t),
\end{aligned}
$$$$
\left(x_{p}^{J}(t)\right)^{\Delta}=-c_{p}(t) x_{p}^{J}\left(t-\delta_{p}(t)\right)
$$$$
+\sum_{q=1}^{n}\left(a_{p q}^{R}(t) f_{q}^{J}\left(x_{q}^{J}\left(t-\tau_{p q}(t)\right)\right)\right.
$$$$
+a_{p q}^{J}(t) f_{q}^{R}\left(x_{q}^{R}\left(t-\tau_{p q}(t)\right)\right)
$$$$
-a_{p q}^{I}(t) f_{q}^{K}\left(x_{q}^{K}\left(t-\tau_{p q}(t)\right)\right)
$$$$
\left.+a_{p q}^{K}(t) f_{q}^{I}\left(x_{q}^{I}\left(t-\tau_{p q}(t)\right)\right)\right)
$$$$
+\sum_{q=1}^{n}\left(b_{p q}^{R}(t) g_{q}^{J}\left(\left(x_{q}^{J}\right)^{\Delta}\left(t-\eta_{p q}(t)\right)\right)\right.
$$$$
+b_{p q}^{J}(t) g_{q}^{R}\left(\left(x_{q}^{R}\right)^{\Delta}\left(t-\eta_{p q}(t)\right)\right)
$$$$
-b_{p q}^{I}(t) g_{q}^{K}\left(\left(x_{q}^{K}\right)^{\Delta}\left(t-\eta_{p q}(t)\right)\right)
$$$$
\left.+b_{p q}^{K}(t) g_{q}^{I}\left(\left(x_{q}^{I}\right)^{\Delta}\left(t-\eta_{p q}(t)\right)\right)\right)+u_{p}^{J}(t),
$$$$
\left(x_{p}^{K}(t)\right)^{\Delta}=-c_{p}(t) x_{p}^{I}\left(t-\delta_{p}(t)\right)
$$$$
+\sum_{q=1}^{n}\left(a_{p q}^{R}(t) f_{q}^{K}\left(x_{q}^{K}\left(t-\tau_{p q}(t)\right)\right)\right.
$$$$
+a_{p q}^{K}(t) f_{q}^{R}\left(x_{q}^{R}\left(t-\tau_{p q}(t)\right)\right)
$$$$
+a_{p q}^{I}(t) f_{q}^{J}\left(x_{q}^{J}\left(t-\tau_{p q}(t)\right)\right)
$$$$
\left.-a_{p q}^{J}(t) f_{q}^{I}\left(x_{q}^{I}\left(t-\tau_{p q}(t)\right)\right)\right)
$$$$
+\sum_{q=1}^{n}\left(b_{p q}^{R}(t) g_{q}^{K}\left(\left(x_{q}^{K}\right)^{\Delta}\left(t-\eta_{p q}(t)\right)\right)\right.
$$$$
+b_{p q}^{K}(t) g_{q}^{R}\left(\left(x_{q}^{R}\right)^{\Delta}\left(t-\eta_{p q}(t)\right)\right)
$$$$
+b_{p q}^{I}(t) g_{q}^{J}\left(\left(x_{q}^{J}\right)^{\Delta}\left(t-\eta_{p q}(t)\right)\right)
$$$$
\left.-b_{p q}^{J}(t) g_{q}^{I}\left(\left(x_{q}^{I}\right)^{\Delta}\left(t-\eta_{p q}(t)\right)\right)\right)+u_{p}^{K}(t),
$$

$a_{p q}(t)=a_{p q}^{R}(t)+i a_{p q}^{I}(t)+j a_{p q}^{J}(t)+k a_{p q}^{K}(t)$,

$b_{p q}(t)=b_{p q}^{R}(t)+i b_{p q}^{I}(t)+j b_{p q}^{J}(t)+k b_{p q}^{K}(t)$,

$u_{p}(t)=u_{p}^{R}(t)+i u_{p}^{I}(t)+j u_{p}^{J}(t)+k u_{p}^{K}(t)$, 
According to (15), we can get

$$
\begin{aligned}
X_{p}^{\Delta}(t)= & -c_{p}(t) X_{p}\left(t-\delta_{p}(t)\right) \\
& +\sum_{q=1}^{n} A_{p q}(t) F_{q}\left(X_{q}\left(t-\tau_{p q}(t)\right)\right) \\
& +\sum_{q=1}^{n} B_{p q}(t) G_{q}\left(X_{q}^{\Delta}\left(t-\eta_{p q}(t)\right)\right)+U_{p}(t),
\end{aligned}
$$

$$
p \in \Lambda
$$

where

$$
\begin{aligned}
& A_{p q}(t)=\left(\begin{array}{cccc}
a_{p q}^{R}(t) & -a_{p q}^{I}(t) & -a_{p q}^{J}(t) & -a_{p q}^{K}(t) \\
a_{p q}^{I}(t) & a_{p q}^{R}(t) & -a_{p q}^{K}(t) & a_{p q}^{J}(t) \\
a_{p q}^{J}(t) & a_{p q}^{K}(t) & a_{p q}^{R}(t) & -a_{p q}^{I}(t) \\
a_{p q}^{K}(t) & -a_{p q}^{J}(t) & a_{p q}^{I}(t) & a_{p q}^{R}(t)
\end{array}\right), \\
& B_{p q}(t)=\left(\begin{array}{cccc}
b_{p q}^{R}(t) & -b_{p q}^{I}(t) & -b_{p q}^{J}(t) & -b_{p q}^{K}(t) \\
b_{p q}^{I}(t) & b_{p q}^{R}(t) & -b_{p q}^{K}(t) & b_{p q}^{J}(t) \\
b_{p q}^{J}(t) & b_{p q}^{K}(t) & b_{p q}^{R}(t) & -b_{p q}^{I}(t) \\
b_{p q}^{K}(t) & -b_{p q}^{J}(t) & b_{p q}^{I}(t) & b_{p q}^{R}(t)
\end{array}\right) \text {, } \\
& X_{p}(t)=\left(x_{p}^{R}(t), x_{p}^{I}(t), x_{p}^{J}(t), x_{p}^{K}(t)\right)^{T} \text {, } \\
& U_{p}(t)=\left(u_{p}^{R}(t), u_{p}^{I}(t), u_{p}^{J}(t), u_{p}^{K}(t)\right)^{T}, \\
& F_{q}=\left(f_{q}^{R}, f_{q}^{I}, f_{q}^{J}, f_{q}^{K}\right)^{T}, \\
& G_{q}=\left(g_{q}^{R}, g_{q}^{I}, g_{q}^{J}, g_{q}^{K}\right)^{T} .
\end{aligned}
$$

The initial condition associated with (17) is of the form

$$
X_{p}(s)=\Phi_{p}(s), \quad p \in \Lambda, s \in[-\theta, 0]_{\mathbb{T}},
$$

where $\Phi_{p}(\mathrm{~s})=\left(\phi_{p}^{R}(s), \phi_{p}^{I}(s), \phi_{p}^{J}(s), \phi_{p}^{K}(s)\right), \phi_{p}^{l}(s) \quad \epsilon$ $C\left([-\theta, 0]_{\mathbb{T}}, \mathbb{R}\right), l \in E$.

Remark 10. It is obvious that if $x(t)=$ $\left(x_{1}^{R}(t), x_{1}^{I}(t), x_{1}^{J}(t), x_{1}^{K}(t), x_{2}^{R}(t), x_{2}^{I}(t), x_{2}^{J}(t), x_{2}^{K}(t), \ldots\right.$, $\left.x_{n}^{R}(t), x_{n}^{I}(t), x_{n}^{J}(t), x_{n}^{K}(t)\right)^{T}$ is a solution to system (17), then

$$
y(t)=\left(X_{1}(t), X_{2}(t), \ldots, X_{n}(t)\right)^{T},
$$

where $x_{p}(t)=x_{p}^{R}(t)+i x_{p}^{I}(t)+j x_{p}^{J}(t)+k x_{p}^{K}(t), p=1,2, \ldots, n$ must be a solution to (1). Thus, the problem of finding a pseudo almost periodic solution for (1) reduces to finding one for system (17). For considering the stability of solutions of (1), we just need to consider the stability of solutions of system (17).

\section{Main Results}

In this section, we will study the existence and global exponential stability of pseudo almost periodic solutions of system (17).
Let $\mathbb{X}=\left\{f \mid f, f^{\Delta} \in \operatorname{PAP}\left(\mathbb{T}, \mathbb{R}^{4 n}\right)\right\}$ with the norm $\|f\|_{\mathbb{X}}=\max \left\{\|f\|,\left\|f^{\Delta}\right\|\right\}$, where $\|f\|=\max _{1 \leq h \leq 4 n}\left\{f_{h}^{+}\right\}$, $\left\|f^{\Delta}\right\|=\max _{1 \leq h \leq 4 n}\left\{\left(f_{h}^{\Delta}\right)^{+}\right\}$; then $\mathbb{X}$ is a Banach space.

Throughout this paper, we assume that the following conditions hold:

$\left(H_{2}\right) c_{p} \in C\left(\mathbb{T}, \mathbb{R}^{+}\right)$with $-c_{p} \in \mathscr{R}^{+}$is an almost periodic function, $A_{p q}, B_{p q} \in \operatorname{AP}\left(\mathbb{T}, \mathbb{R}^{4 \times 4}\right), U_{p} \in \operatorname{PAP}(\mathbb{T}$, $\left.\mathbb{R}^{4 \times 1}\right), \delta_{p} \in C(\mathbb{T}, \Pi), \tau_{p q}, \eta_{p q} \in C^{1}(\mathbb{T}, \Pi), \inf _{t \in \mathbb{T}}(1-$ $\left.\tau_{p q}^{\Delta}(t)\right)>0, \inf _{t \in \mathbb{T}}\left(1-\eta_{p q}^{\Delta}(t)\right)>0, p, q \in \Lambda$.

$\left(H_{3}\right)$ Functions $f_{q}^{l}, g_{q}^{l} \in C(\mathbb{R}, \mathbb{R})$ and there exist positive constants $\alpha_{q}^{l}, \beta_{q}^{l}$ such that for all $x^{l}, y^{l} \in \mathbb{R}$

$$
\begin{aligned}
& \left|f_{q}^{l}\left(x^{l}\right)-f_{q}^{l}\left(y^{l}\right)\right| \leq \alpha_{q}^{l}\left|x^{l}-y^{l}\right|, \\
& \left|g_{q}^{l}\left(x^{l}\right)-g_{q}^{l}\left(y^{l}\right)\right| \leq \beta_{q}^{l}\left|x^{l}-y^{l}\right|,
\end{aligned}
$$

and $f_{q}^{l}(0)=g_{q}^{l}(0)=0, q \in \Lambda, l \in E$.

$\left(H_{4}\right)$ There exists a positive constant $\kappa$ such that

$$
\begin{aligned}
& \max _{p \in \Lambda}\left\{\max _{l \in E}\left\{\frac{\Gamma_{p}^{l} \kappa+u_{p}^{l^{+}}}{c_{p}^{-}},\left(1+\frac{c_{p}^{+}}{c_{p}^{-}}\right)\left(\Gamma_{p}^{l} \kappa+u_{p}^{l^{+}}\right)\right\}\right\} \\
& \leq \kappa, \\
& \max _{p \in \Lambda}\left\{\max _{l \in E}\left\{\frac{\Gamma_{p}^{l}}{c_{p}^{-}},\left(1+\frac{c_{p}^{+}}{c_{p}^{-}}\right) \Gamma_{p}^{l}\right\}\right\}:=\rho<1,
\end{aligned}
$$

where

$$
\begin{aligned}
\Gamma_{p}^{l} & =c_{p}^{+} \delta_{p}^{+}+A_{p}^{l}+B_{p}^{l}, \quad p \in \Lambda, l \in E, \\
A_{p}^{R} & =\sum_{q=1}^{n}\left(a_{p q}^{R^{+}} \alpha_{q}^{R}+a_{p q}^{I^{+}} \alpha_{q}^{I}+a_{p q}^{J^{+}} \alpha_{q}^{J}+a_{p q}^{K^{+}} \alpha_{q}^{K}\right),
\end{aligned}
$$

$p \in \Lambda$

$$
B_{p}^{R}=\sum_{q=1}^{n}\left(b_{p q}^{R^{+}} \beta_{q}^{R}+b_{p q}^{I^{+}} \beta_{q}^{I}+b_{p q}^{J^{+}} \beta_{q}^{J}+b_{p q}^{K^{+}} \beta_{q}^{K}\right),
$$

$p \in \Lambda$

$\mathrm{A}_{p}^{I}=\sum_{q=1}^{n}\left(a_{p q}^{R^{+}} \alpha_{q}^{I}+a_{p q}^{I^{+}} \alpha_{q}^{R}+a_{p q}^{J^{+}} \alpha_{q}^{K}+a_{p q}^{K^{+}} \alpha_{q}^{J}\right)$

$p \in \Lambda$

$$
B_{p}^{I}=\sum_{q=1}^{n}\left(b_{p q}^{R^{+}} \beta_{q}^{I}+b_{p q}^{I^{+}} \beta_{q}^{R}+b_{p q}^{J^{+}} \beta_{q}^{K}+b_{p q}^{K^{+}} \beta_{q}^{J}\right),
$$

$p \in \Lambda$ 


$$
\begin{array}{ll}
A_{p}^{J}=\sum_{q=1}^{n}\left(a_{p q}^{R^{+}} \alpha_{q}^{J}+a_{p q}^{J^{+}} \alpha_{q}^{R}+a_{p q}^{I^{+}} \alpha_{q}^{K}+a_{p q}^{K^{+}} \alpha_{q}^{I}\right), & \\
B_{p}^{J}=\sum_{q=1}^{n}\left(b_{p q}^{R^{+}} \beta_{q}^{I}+b_{p q}^{J^{+}} \beta_{q}^{R}+b_{p q}^{I^{+}} \beta_{q}^{K}+b_{p q}^{K^{+}} \beta_{q}^{I}\right), & \\
A_{p}^{K}=\sum_{q=1}^{n}\left(a_{p q}^{R^{+}} \alpha_{q}^{K}+a_{p q}^{K^{+}} \alpha_{q}^{R}+a_{p q}^{I^{+}} \alpha_{q}^{J}+a_{p q}^{J^{+}} \alpha_{q}^{I}\right), & \\
B_{p}^{K}=\sum_{q=1}^{n}\left(b_{p q}^{R^{+}} \beta_{q}^{K}+b_{p q}^{K^{+}} \beta_{q}^{R}+b_{p q}^{I^{+}} \beta_{q}^{J}+b_{p q}^{J^{+}} \beta_{q}^{I}\right), &
\end{array}
$$$$
p \in \Lambda \text {. }
$$

Theorem 11. Assume that $\left(H_{1}\right)-\left(H_{4}\right)$ hold; then system (17) has a unique pseudo almost periodic solution in the region $\mathbb{X}^{*}=\left\{\varphi \in \mathbb{X} \mid\|\varphi\|_{\mathbb{X}} \leq \kappa\right\}$.

Proof. System (17) can be written as

$$
\begin{aligned}
X_{p}^{\Delta}(t)= & -c_{p}(t) X_{p}(t)+c_{p}(t) \int_{t-\delta_{p}(t)}^{t} X_{p}^{\Delta}(s) \Delta s \\
& +\sum_{q=1}^{n} A_{p q}(t) F_{q}\left(X_{q}\left(t-\tau_{p q}(t)\right)\right) \\
& +\sum_{q=1}^{n} B_{p q}(t) G_{q}\left(X_{q}^{\Delta}\left(t-\eta_{p q}(t)\right)\right)+U_{p}(t), \\
& p \in \Lambda, t \in \mathbb{T} .
\end{aligned}
$$

For any $\varphi \in \mathbb{X}$, consider the linear dynamic system

$$
\begin{aligned}
X_{p}^{\Delta}(t)= & -c_{p}(t) X_{p}(t)+c_{p}(t) \int_{t-\delta_{p}(t)}^{t} \varphi_{p}^{\Delta}(s) \Delta s \\
& +\sum_{q=1}^{n} A_{p q}(t) F_{q}\left(\varphi_{q}\left(t-\tau_{p q}(t)\right)\right)
\end{aligned}
$$

$$
+\sum_{q=1}^{n} B_{p q}(t) G_{q}\left(\varphi_{q}^{\Delta}\left(t-\eta_{p q}(t)\right)\right)+U_{p}(t)
$$

$p \in \Lambda, t \in \mathbb{T}$.

Since $\min _{1 \leq p \leq n}\left\{\inf _{t \in \mathbb{T}} c_{p}(t)\right\}>0$ and $-c_{p} \in \mathscr{R}^{+}$, it follows from Lemma 9 that the linear system

$$
X_{p}^{\Delta}(t)=-c_{p}(t) X_{p}(t)
$$

admits an exponential dichotomy on $\mathbb{T}$. Thus, by Lemma 8 , we see that system (25) has exactly one pseudo almost periodic solution which can be expressed as follows:

$$
\begin{array}{r}
X_{p}^{\varphi}(t)=\int_{-\infty}^{t} e_{-c_{p}}(t, \sigma(s))\left(c_{p}(s) \int_{s-\delta_{p}(s)}^{s} \varphi_{p}^{\Delta}(u) \Delta u\right. \\
+\sum_{q=1}^{n} A_{p q}(s) F_{q}\left(\varphi_{q}\left(s-\tau_{p q}(s)\right)\right) \\
\left.+\sum_{q=1}^{n} B_{p q}(s) G_{q}\left(\varphi_{q}^{\Delta}\left(s-\eta_{p q}(s)\right)\right)+U_{p}(s)\right) \Delta s, \\
p \in \Lambda .
\end{array}
$$

Now, we define the operator $\Phi: \mathbb{X}^{*} \rightarrow \mathbb{X}^{*}$ as

$$
\left(\varphi_{1}, \varphi_{2}, \ldots, \varphi_{n}\right)^{T} \longrightarrow\left(X_{1}^{\varphi}, X_{2}^{\varphi}, \ldots, X_{n}^{\varphi}\right)^{T}
$$

where $\varphi_{p}=\left(\varphi_{p}^{R}, \varphi_{p}^{I}, \varphi_{p}^{J}, \varphi_{p}^{K}\right), X_{p}^{\varphi}$ is defined by (27), $p \in \Lambda$.

First, we show that, for any $\varphi \in \mathbb{X}^{*}$, we have $\Phi \varphi \in \mathbb{X}^{*}$. From (27), we have

$$
\begin{aligned}
& \left|(\Phi \varphi)_{p}^{R}(t)\right|=\mid \int_{-\infty}^{t} e_{-c_{p}}(t, \sigma(s))\left(c_{p}(s) \int_{s-\delta_{p}(s)}^{s}\left(\varphi_{p}^{R}\right)^{\Delta}(u) \Delta u\right. \\
& \quad+\sum_{q=1}^{n}\left(a_{p q}^{R}(s) f_{q}^{R}\left(\varphi_{q}^{R}\left(s-\tau_{p q}(s)\right)\right)-a_{p q}^{I}(s) f_{q}^{I}\left(\varphi_{q}^{I}\left(s-\tau_{p q}(s)\right)\right)-a_{p q}^{J}(s) f_{q}^{J}\left(\varphi_{q}^{J}\left(s-\tau_{p q}(s)\right)\right)-a_{p q}^{K}(s) f_{q}^{K}\left(\varphi_{q}^{K}\left(s-\tau_{p q}(s)\right)\right)\right) \\
& \quad+\sum_{q=1}^{n}\left(b_{p q}^{R}(s) g_{q}^{R}\left(\left(\varphi_{q}^{R}\right)^{\Delta}\left(s-\eta_{p q}(s)\right)\right)-b_{p q}^{I}(s) g_{q}^{I}\left(\left(\varphi_{q}^{I}\right)^{\Delta}\left(s-\eta_{p q}(s)\right)\right)-b_{p q}^{J}(s) g_{q}^{J}\left(\left(\varphi_{q}^{J}\right)^{\Delta}\left(s-\eta_{p q}(s)\right)\right)-b_{p q}^{K}(s) g_{q}^{K}\left(\left(\varphi_{q}^{K}\right)^{\Delta}\left(s-\eta_{p q}(s)\right)\right)\right) \\
& \left.\quad+u_{p}^{R}(s)\right) \Delta s \mid \leq \int_{-\infty}^{t} e_{-c_{p}}(t, \sigma(s))\left(c_{p}^{+} \int_{s-\delta_{p}(s)}^{s}\left|\left(\varphi_{p}^{R}\right)^{\Delta}(u)\right| \Delta u\right.
\end{aligned}
$$




$$
\begin{aligned}
& +\sum_{q=1}^{n}\left(a_{p q}^{R^{+}}\left|f_{q}^{R}\left(\varphi_{q}^{R}\left(s-\tau_{p q}(s)\right)\right)\right|+a_{p q}^{I^{+}}\left|f_{q}^{I}\left(\varphi_{q}^{I}\left(s-\tau_{p q}(s)\right)\right)\right|+a_{p q}^{J^{+}}\left|f_{q}^{J}\left(\varphi_{q}^{J}\left(s-\tau_{p q}(s)\right)\right)\right|+a_{p q}^{K^{+}}\left|f_{q}^{K}\left(\varphi_{q}^{K}\left(s-\tau_{p q}(s)\right)\right)\right|\right) \\
& +\sum_{q=1}^{n}\left(b_{p q}^{R^{+}}\left|g_{q}^{R}\left(\left(\varphi_{q}^{R}\right)^{\Delta}\left(s-\eta_{p q}(s)\right)\right)\right|+b_{p q}^{I^{+}}\left|g_{q}^{I}\left(\left(\varphi_{q}^{I}\right)^{\Delta}\left(s-\eta_{p q}(s)\right)\right)\right|+b_{p q}^{J^{+}}\left|g_{q}^{J}\left(\left(\varphi_{q}^{J}\right)^{\Delta}\left(s-\eta_{p q}(s)\right)\right)\right|+b_{p q}^{K^{+}}\left|g_{q}^{K}\left(\left(\varphi_{q}^{K}\right)^{\Delta}\left(s-\eta_{p q}(s)\right)\right)\right|\right) \\
& \left.+u_{p}^{R^{+}}\right) \Delta s \leq \int_{-\infty}^{t} e_{-c_{p}}(t, \sigma(s))\left(c_{p}^{+} \delta_{p}^{+} \kappa+\sum_{q=1}^{n}\left(a_{p q}^{R^{+}} \alpha_{q}^{R} \kappa+a_{p q}^{I^{+}} \alpha_{q}^{I} \kappa+a_{p q}^{J^{+}} \alpha_{q}^{J} \kappa+a_{p q}^{K^{+}} \alpha_{q}^{K} \kappa\right)+\sum_{q=1}^{n}\left(b_{p q}^{R^{+}} \beta_{q}^{R} \kappa+b_{p q}^{I^{+}} \beta_{q}^{I} \kappa+b_{p q}^{J^{+}} \beta_{q}^{J} \kappa+b_{p q}^{K^{+}} \beta_{q}^{K} \kappa\right)\right. \\
& \left.+u_{p}^{R^{+}}\right) \Delta s \leq \frac{1}{c_{p}^{-}}\left(c_{p}^{+} \delta_{p}^{+} \kappa+A_{p}^{R} \kappa+B_{p}^{R} \kappa+u_{p}^{R^{+}}\right)=\frac{\Gamma_{p}^{R} \kappa+u_{p}^{R^{+}}}{c_{p}^{-}}, \quad p \in \Lambda .
\end{aligned}
$$

In a similar way, we have

On the other hand, we have

$$
\left|(\Phi \varphi)_{p}^{l}(t)\right| \leq \frac{\Gamma_{p}^{l} \kappa+u_{p}^{l^{+}}}{c_{p}^{-}}, \quad p \in \Lambda, l=I, J, K .
$$

$$
\begin{aligned}
& \left|\left((\Phi \varphi)_{p}^{R}\right)^{\Delta}(t)\right|=\mid c_{p}(t) \int_{t-\delta_{p}(t)}^{t}\left(\varphi_{p}^{R}\right)^{\Delta}(u) \Delta u+\sum_{q=1}^{n}\left(a_{p q}^{R}(t) f_{q}^{R}\left(\varphi_{q}^{R}\left(t-\tau_{p q}(t)\right)\right)-a_{p q}^{I}(t) f_{q}^{I}\left(\varphi_{q}^{I}\left(t-\tau_{p q}(t)\right)\right)-a_{p q}^{J}(t) f_{q}^{J}\left(\varphi_{q}^{J}\left(t-\tau_{p q}(t)\right)\right)\right. \\
& \left.\quad-a_{p q}^{K}(t) f_{q}^{K}\left(\varphi_{q}^{K}\left(t-\tau_{p q}(t)\right)\right)\right)+\sum_{q=1}^{n}\left(b_{p q}^{R}(t) g_{q}^{R}\left(\left(\varphi_{q}^{R}\right)^{\Delta}\left(t-\eta_{p q}(t)\right)\right)-b_{p q}^{I}(t) g_{q}^{I}\left(\left(\varphi_{q}^{I}\right)^{\Delta}\left(t-\eta_{p q}(t)\right)\right)-b_{p q}^{J}(t) g_{q}^{J}\left(\left(\varphi_{q}^{J}\right)^{\Delta}\left(t-\eta_{p q}(t)\right)\right)\right. \\
& \left.\quad-b_{p q}^{K}(t) g_{q}^{K}\left(\left(\varphi_{q}^{K}\right)^{\Delta}\left(t-\eta_{p q}(t)\right)\right)\right)+u_{p}^{R}(t)-c_{p}(t) \int_{-\infty}^{t} e_{-c_{p}}(t, \sigma(s))\left(c_{p}(s) \int_{s-\delta_{p}(s)}^{s}\left(\varphi_{p}^{R}\right)^{\Delta}(u) \Delta u\right. \\
& \quad+\sum_{q=1}^{n}\left(a_{p q}^{R}(s) f_{q}^{R}\left(\varphi_{q}^{R}\left(s-\tau_{p q}(s)\right)\right)-a_{p q}^{I}(s) f_{q}^{I}\left(\varphi_{q}^{I}\left(s-\tau_{p q}(s)\right)\right)-a_{p q}^{J}(s) f_{q}^{J}\left(\varphi_{q}^{J}\left(s-\tau_{p q}(s)\right)\right)-a_{p q}^{K}(s) f_{q}^{K}\left(\varphi_{q}^{K}\left(s-\tau_{p q}(s)\right)\right)\right) \\
& \quad+\sum_{q=1}^{n}\left(b_{p q}^{R}(s) g_{q}^{R}\left(\left(\varphi_{q}^{R}\right)^{\Delta}\left(s-\eta_{p q}(s)\right)\right)-b_{p q}^{I}(s) g_{q}^{I}\left(\left(\varphi_{q}^{I}\right)^{\Delta}\left(s-\eta_{p q}(s)\right)\right)-b_{p q}^{J}(s) g_{q}^{J}\left(\left(\varphi_{q}^{J}\right)^{\Delta}\left(s-\eta_{p q}(s)\right)\right)-b_{p q}^{K}(s) g_{q}^{K}\left(\left(\varphi_{q}^{K}\right)^{\Delta}\left(s-\eta_{p q}(s)\right)\right)\right) \\
& \left.\quad+u_{p}^{R}(s)\right) \Delta s \mid \leq\left(1+\frac{c_{p}^{+}}{c_{p}^{-}}\right)\left(c_{p}^{+} \delta_{p}^{+} \kappa+A_{p}^{R} \kappa+B_{p}^{R} \kappa+u_{p}^{R^{+}}\right)=\left(1+\frac{c_{p}^{+}}{c_{p}^{-}}\right)\left(\Gamma_{p}^{R} \kappa+u_{p}^{R^{+}}\right), \quad p \in \Lambda .
\end{aligned}
$$

In a similar way, we have

$$
\begin{array}{r}
\left|\left((\Phi \varphi)_{p}^{l}\right)^{\Delta}(t)\right| \leq\left(1+\frac{c_{p}^{+}}{c_{p}^{-}}\right)\left(\Gamma_{p}^{l} \kappa+u_{p}^{l^{+}}\right), \\
\quad p \in \Lambda, l=I, J, K .
\end{array}
$$

It follows from (29) to $(32)$ and $\left(H_{4}\right)$ that

$$
\|\Phi \varphi\|_{\mathbb{X}} \leq \kappa
$$

which implies that $\Phi \varphi \in \mathbb{X}^{*}$, so the mapping $\Phi$ is a selfmapping from $\mathbb{X}^{*}$ to $\mathbb{X}^{*}$. Next, we shall prove that $\Phi$ is a contraction mapping. In fact, for any $\varphi, \psi \in \mathbb{X}^{*}$, we have

$$
\begin{aligned}
& \left|(\Phi \varphi-\Phi \psi)_{p}^{R}(t)\right| \\
& \quad=\mid \int_{-\infty}^{t} e_{-c_{p}}(t, \sigma(s))
\end{aligned}
$$

$$
\begin{aligned}
& \cdot\left(c_{p}(s) \int_{s-\delta_{p}(s)}^{s}\left(\left(\varphi_{p}^{R}\right)^{\Delta}(u)-\left(\psi_{p}^{R}\right)^{\Delta}(u)\right) \Delta u\right. \\
& +\sum_{q=1}^{n}\left(a_{p q}^{R}(s)\left(f_{q}^{R}\left(\varphi_{q}^{R}\left(s-\tau_{p q}(s)\right)\right)-f_{q}^{R}\left(\psi_{q}^{R}\left(s-\tau_{p q}(s)\right)\right)\right)\right. \\
& -a_{p q}^{I}(s)\left(f_{q}^{I}\left(\varphi_{q}^{I}\left(s-\tau_{p q}(s)\right)\right)-f_{q}^{I}\left(\psi_{q}^{I}\left(s-\tau_{p q}(s)\right)\right)\right) \\
& -a_{p q}^{J}(s)\left(f_{q}^{J}\left(\varphi_{q}^{J}\left(s-\tau_{p q}(s)\right)\right)-f_{q}^{J}\left(\psi_{q}^{J}\left(s-\tau_{p q}(s)\right)\right)\right) \\
& \left.-a_{p q}^{K}(s)\left(f_{q}^{K}\left(\varphi_{q}^{K}\left(s-\tau_{p q}(s)\right)\right)-f_{q}^{K}\left(\psi_{q}^{K}\left(s-\tau_{p q}(s)\right)\right)\right)\right) \\
& +\sum_{q=1}^{n}\left(b_{p q}^{R}(s)\left(g_{q}^{R}\left(\left(\varphi_{q}^{R}\right)^{\Delta}\left(s-\eta_{p q}(s)\right)\right)-g_{q}^{R}\left(\left(\psi_{q}^{R}\right)^{\Delta}\left(s-\eta_{p q}(s)\right)\right)\right)\right. \\
& -b_{p q}^{I}(s)\left(g_{q}^{I}\left(\left(\varphi_{q}^{I}\right)^{\Delta}\left(s-\eta_{p q}(s)\right)\right)-g_{q}^{I}\left(\left(\psi_{q}^{I}\right)^{\Delta}\left(s-\eta_{p q}(s)\right)\right)\right) \\
& -b_{p q}^{J}(s)\left(g_{q}^{J}\left(\left(\varphi_{q}^{J}\right)^{\Delta}\left(s-\eta_{p q}(s)\right)\right)-g_{q}^{J}\left(\left(\psi_{q}^{J}\right)^{\Delta}\left(s-\eta_{p q}(s)\right)\right)\right) \\
& \left.\left.-b_{p q}^{K}(s)\left(g_{q}^{K}\left(\left(\varphi_{q}^{K}\right)^{\Delta}\left(s-\eta_{p q}(s)\right)\right)-g_{q}^{K}\left(\left(\psi_{q}^{K}\right)^{\Delta}\left(s-\eta_{p q}(s)\right)\right)\right)\right)\right) \Delta s
\end{aligned}
$$




$$
\begin{aligned}
& \leq \frac{1}{c_{p}^{-}}\left(c_{p}^{+} \delta_{p}^{+}+\sum_{q=1}^{n}\left(a_{p q}^{R^{+}} \alpha_{q}^{R}+a_{p q}^{I^{+}} \alpha_{q}^{I}+a_{p q}^{I^{+}} \alpha_{q}^{J}+a_{p q}^{K^{+}} \alpha_{q}^{K}\right.\right. \\
& \left.\left.+b_{p q}^{R^{+}} \beta_{q}^{R}+b_{p q}^{I^{+}} \beta_{q}^{I}+b_{p q}^{J^{+}} \beta_{q}^{J}+b_{p q}^{K^{+}} \beta_{q}^{K}\right)\right) \\
& \cdot\|\varphi-\psi\|_{\mathbb{X}}=\frac{\Gamma_{p}^{R}}{c_{p}^{-}}\|\varphi-\psi\|_{\mathbb{X}}, \quad p \in \Lambda .
\end{aligned}
$$

In a similar way, we have

$$
\left|(\Phi \varphi-\Phi \psi)_{p}^{l}(t)\right| \leq \frac{\Gamma_{p}^{l}}{c_{p}^{+}}\|\varphi-\psi\|_{\mathbb{X}}
$$

$$
p \in \Lambda, l=I, J, K
$$

On the other hand, we have

$$
\begin{aligned}
& \left|\left((\Phi \varphi-\Phi \psi)_{h}^{R}\right)^{\Delta}(t)\right| \leq\left(c_{p}^{+} \delta_{p}^{+}+\sum_{q=1}^{n}\left(a_{p q}^{R^{+}} \alpha_{q}^{R}+a_{p q}^{I^{+}} \alpha_{q}^{I}\right.\right. \\
& +a_{p q}^{J^{+}} \alpha_{q}^{J}+a_{p q}^{K^{+}} \alpha_{q}^{K}+b_{p q}^{R^{+}} \beta_{q}^{R}+b_{p q}^{I^{+}} \beta_{q}^{I}+b_{p q}^{J^{+}} \beta_{q}^{J} \\
& \left.\left.+b_{p q}^{K^{+}} \beta_{q}^{K}\right)\right)\|\varphi-\psi\|_{\mathbb{X}}+\frac{c_{p}^{+}}{c_{p}^{-}}\left(c_{p}^{+} \delta_{p}^{+}+\sum_{q=1}^{n}\left(a_{p q}^{R^{+}} \alpha_{q}^{R}\right.\right. \\
& +a_{p q}^{I^{+}} \alpha_{q}^{I}+a_{p q}^{J^{+}} \alpha_{q}^{J}+a_{p q}^{K^{+}} \alpha_{q}^{K}+b_{p q}^{R^{+}} \beta_{q}^{R}+b_{p q}^{I^{+}} \beta_{q}^{I} \\
& \left.\left.+b_{p q}^{J^{+}} \beta_{q}^{J}+b_{p q}^{K^{+}} \beta_{q}^{K}\right)\right)\|\varphi-\psi\|_{\mathbb{X}}=\left(1+\frac{c_{p}^{+}}{c_{p}^{-}}\right) \Gamma_{p}^{R} \| \varphi \\
& \quad-\psi \|_{\mathbb{X}}, \quad p \in \Lambda .
\end{aligned}
$$

In a similar way, we have

$$
\begin{array}{r}
\left|\left((\Phi \varphi-\Phi \psi)_{p}^{l}\right)^{\Delta}(t)\right| \leq\left(1+\frac{c_{p}^{+}}{c_{p}^{-}}\right) \Gamma_{p}^{l}\|\varphi-\psi\|_{\mathbb{X}}, \\
p \in \Lambda, l=I, J, K .
\end{array}
$$

From (34) to (37) and $\left(H_{4}\right)$ it follows that

$$
\|\Phi \varphi-\Phi \psi\|_{X} \leq \rho\|\varphi-\psi\|_{X} .
$$

Hence, we obtain that $\Phi$ is a contraction mapping. Then, system (17) has a unique pseudo almost periodic solution in the region $\mathbb{X}^{*}=\left\{\varphi \in \mathbb{X}:\|\varphi\|_{\mathbb{X}} \leq \kappa\right\}$. The proof is complete.

Theorem 12. Assume that $\left(H_{1}\right)-\left(H_{4}\right)$ hold; then system (17) has a unique pseudo almost periodic solution that is globally exponentially stable.

Proof. From Theorem 11, we see that system (17) has a pseudo almost periodic solution $X^{*}(t)=\left(X_{1}^{*}(t), X_{2}^{*}(t), \ldots, X_{n}^{*}(t)\right)^{T}$ with initial value $\Phi^{*}(s)=\left(\varphi_{1}^{*}(t), \varphi_{2}^{*}(t), \ldots, \varphi_{n}^{*}(t)\right)^{T}$. Suppose that $X(t)=\left(X_{1}(t), X_{2}(t), \ldots, X_{n}(t)\right)^{T}$ is an arbitrary solution of system (17) with initial value $\Phi(s)=\left(\varphi_{1}(t)\right.$, $\left.\varphi_{2}(t), \ldots, \varphi_{n}(t)\right)^{T}$ and let $Z(t)=X(t)-X^{*}(t)$; then we have

$$
\begin{aligned}
& Z_{p}^{\Delta}(t)=\left\{-a_{p}(t)\left(X_{p}\left(t-\delta_{p}(t)\right)-X_{p}^{*}\left(t-\delta_{p}(t)\right)\right)\right. \\
& +\sum_{q=1}^{n} A_{p q}(t)\left(F_{q}\left(X_{q}\left(t-\tau_{p q}(t)\right)\right)\right. \\
& \left.-F_{q}\left(X_{q}^{*}\left(t-\tau_{p q}(t)\right)\right)\right)+\sum_{q=1}^{n} B_{p q}(t) \\
& \cdot\left(F_{q}\left(X_{q}^{\Delta}\left(t-\eta_{p q}(t)\right)\right)\right. \\
& \left.\left.-F_{q}\left(\left(X_{q}^{*}\right)^{\Delta}\left(t-\eta_{p q}(t)\right)\right)\right)\right\}, \quad p \in \Lambda \text {. } \\
& \Theta_{p}^{l}(\zeta)=c_{p}^{-}-\zeta-\exp \left(\zeta \sup _{t \in \mathbb{T}} \mu(s)\right)\left(c_{p}^{+} \delta_{p}^{+} \exp \left(\zeta \delta_{p}^{+}\right)\right. \\
& \left.+A_{p}^{l} \exp \left(\zeta \tau_{p q}^{+}\right)+B_{p}^{l} \exp \left(\zeta \eta_{p q}^{+}\right)\right) \\
& \Pi_{p}^{l}(\zeta)=c_{p}^{-}-\zeta-\left(c_{p}^{+} \exp \left(\zeta \sup _{t \in \mathbb{U}} \mu(s)\right)+c_{p}^{-}-\zeta\right) \\
& \cdot\left(c_{p}^{+} \delta_{p}^{+} \exp \left(\zeta \delta_{p}^{+}\right)+A_{p}^{l} \exp \left(\zeta \tau_{p q}^{+}\right)\right. \\
& \left.+B_{p}^{l} \exp \left(\zeta \eta_{p q}^{+}\right)\right) \text {. }
\end{aligned}
$$

By $\left(H_{4}\right)$, we have

$$
\begin{aligned}
& \Theta_{p}^{l}(0)=c_{p}^{-}-\Gamma_{p}^{l}>0, \\
& \Pi_{p}^{l}(0)=c_{p}^{-}-\left(c_{p}^{+}+c_{p}^{-}\right) \Gamma_{p}^{l}>0,
\end{aligned}
$$

$p \in \Lambda, l \in E$.

Since $\Theta_{p}^{l}$ and $\Pi_{p}^{l}$ are continuous on $[0,+\infty)$ and $\Theta_{p}^{l}(\zeta), \Pi_{p}^{l}(\zeta) \rightarrow-\infty$, as $\zeta \rightarrow+\infty$, there exist $\xi_{p}{ }^{l}, \xi_{p}^{* l}>0$ such that $\Theta_{p}^{l}\left(\xi_{p}{ }^{l}\right)=\Pi_{p}^{l}\left(\xi_{p}^{*}\right)=0$ and $\Theta_{p}^{l}(\zeta)>0$ for $\zeta \in\left(0, \xi_{p}{ }^{l}\right), \Pi_{p}^{l}(\zeta)>0$ for $\zeta \in\left(0, \xi_{p}^{* l}\right), p \in \Lambda, l \in E$. Take $\gamma=\min _{p \in \Lambda, l \in E}\left\{\xi_{p}{ }^{l}, \xi_{p}^{* l}\right\}$; we have $\Theta_{p}^{l}(\gamma) \geq 0, \Pi_{p}^{l}(\gamma) \geq 0$. So, we can choose a positive constant $0<\lambda<\min \left\{\gamma, \min _{p \in \Lambda}\left\{c_{p}^{-}\right\}\right\}$ such that

$$
\begin{aligned}
& \Theta_{p}^{l}(\lambda)>0, \\
& \Pi_{p}^{l}(\lambda)>0,
\end{aligned}
$$


which imply that, for $p \in \Lambda, l \in E$,

$$
\begin{aligned}
& \frac{\exp \left(\lambda \sup _{t \in \mathbb{T}} \mu(s)\right)}{c_{p}^{-}-\lambda}\left(c_{p}^{+} \delta_{p}^{+} \exp \left(\lambda \delta_{p}^{+}\right)\right. \\
& \left.+A_{p}^{l} \exp \left(\lambda \tau_{p q}^{+}\right)+B_{p}^{l} \exp \left(\lambda \eta_{p q}^{+}\right)\right)<1, \\
& \left(1+\frac{c_{p}^{+} \exp \left(\lambda \sup _{t \in \mathbb{T}} \mu(s)\right)}{c_{p}^{-}-\lambda}\right)\left(c_{p}^{+} \delta_{p}^{+} \exp \left(\lambda \delta_{p}^{+}\right)\right. \\
& \left.+A_{p}^{l} \exp \left(\lambda \tau_{p q}^{+}\right)+B_{p}^{l} \exp \left(\lambda \eta_{p q}^{+}\right)\right)<1 .
\end{aligned}
$$

Let $M=\max _{p \in \Lambda} \max _{l \in E}\left\{c_{p}^{-} / \Gamma_{p}^{l}\right\}$; then by $\left(H_{4}\right)$ we have $M>1$. Thus,

$$
\begin{gathered}
\frac{1}{M}-\min _{p \in \Lambda, l \in E}\left\{\frac { \operatorname { e x p } ( \lambda \operatorname { s u p } _ { t \in \mathbb { T } } \mu ( s ) ) } { c _ { p } ^ { - } - \lambda } \left(c_{p}^{+} \delta_{p}^{+} \exp \left(\lambda \delta_{p}^{+}\right)\right.\right. \\
\left.\left.+A_{p}^{l} \exp \left(\lambda \tau_{p q}^{+}\right)+B_{p}^{l} \exp \left(\lambda \eta_{p q}^{+}\right)\right)\right\}<0 .
\end{gathered}
$$

Let

$$
\begin{aligned}
\|Z(t)\| & =\max _{p \in \Lambda}\left\{\max _{l \in E}\left\{\left|x_{p}^{l}(t)-x_{p}^{* l}(t)\right|,\left|\left(x_{p}^{l}\right)^{\Delta}(t)-\left(x_{p}^{* l}\right)^{\Delta}(t)\right|\right\}\right\}, \\
\|\phi\|_{0} & =\max _{1 \leq p \leq n}\left\{\max _{l \in E}\left\{\sup _{s \in[-\theta, 0]_{\top}}\left|\varphi_{p}^{l}(s)-\varphi_{p}^{* l}(s)\right|, \sup _{s \in[-\theta, 0]_{\top}}\left|\left(\varphi_{p}^{l}\right)^{\Delta}(s)-\left(\varphi_{p}^{* l}\right)^{\Delta}(s)\right|\right\}\right\} .
\end{aligned}
$$

Then, for any $\epsilon>0$, it is obvious that

$$
\begin{aligned}
\|Z(0)\| & <\left(\|\phi\|_{0}+\epsilon\right), \\
\|Z(t)\| & <M\left(\|\phi\|_{0}+\epsilon\right) e_{\ominus \lambda}(t, 0), \quad \forall t \in[-\theta, 0]_{\mathbb{T}} .
\end{aligned}
$$

We claim that

$$
\|Z(t)\|<M\left(\|\phi\|_{0}+\epsilon\right) e_{\ominus \lambda}(t, 0), \quad \forall t \in(0,+\infty)_{\mathbb{T}} .
$$

If (48) is not true, then there must be some $t_{1} \in(0,+\infty)_{\mathbb{T}}$ such that

$$
\begin{aligned}
\left\|Z\left(t_{1}\right)\right\| & \geq M\left(\|\phi\|_{0}+\epsilon\right) e_{\ominus \lambda}\left(t_{1}, 0\right), \\
\|Z(t)\| & <M\left(\|\phi\|_{0}+\epsilon\right) e_{\ominus \lambda}(t, 0), \quad t \in\left[-\theta, t_{1}\right)_{\mathbb{T}} .
\end{aligned}
$$

Therefore, there must exist a constant $c \geq 1$ such that

$$
\begin{gathered}
\left\|Z\left(t_{1}\right)\right\|=c M\left(\|\phi\|_{0}+\epsilon\right) e_{\ominus \lambda}\left(t_{1}, 0\right), \\
\|Z(t)\|<c M\left(\|\phi\|_{0}+\epsilon\right) e_{\ominus \lambda}(t, 0),
\end{gathered}
$$

$$
t \in\left[-\theta, t_{1}\right)_{\mathbb{T}} .
$$

Multiplying the both sides of (39) by $e_{-c_{p}}(0, \sigma(t))$ and integrating over $[0, t]_{\mathbb{T}}$, we get

$$
\begin{aligned}
& Z_{p}(t)=\left\{Z_{p}(0) e_{-c_{p}}(t, 0)+\int_{0}^{t} e_{-c_{p}}(t, \sigma(s))\left(a_{p}(s) \int_{s-\delta_{p}(s)}^{s} Z_{p}^{\Delta}(u) \Delta u\right.\right. \\
& \quad+\sum_{q=1}^{n} A_{p q}(s)\left(F_{q}\left(X_{q}\left(s-\tau_{p q}(s)\right)\right)-F_{q}\left(X_{q}^{*}\left(s-\tau_{p q}(s)\right)\right)\right) \\
& \left.\left.\quad+\sum_{q=1}^{n} B_{p q}(s)\left(F_{q}\left(X_{q}^{\Delta}\left(s-\eta_{p q}(s)\right)\right)-F_{q}\left(\left(X_{q}^{*}\right)^{\Delta}\left(s-\eta_{p q}(s)\right)\right)\right)\right) \Delta s\right\}, \quad p \in \Lambda .
\end{aligned}
$$

In view of (46), (47), and (50) and $M>1$, we have

$$
\begin{aligned}
\left|\left(x-x^{*}\right)_{p}^{R}\left(t_{1}\right)\right| \\
\quad=\mid\left(x_{p}^{R}(0)-x_{p}^{* R}(0)\right) e_{-c_{p}}\left(t_{1}, 0\right) \\
\quad+\int_{0}^{t_{1}} e_{-c_{p}}\left(t_{1}, \sigma(s)\right)
\end{aligned}
$$

$$
\begin{aligned}
& \cdot\left(c_{p}(s) \int_{s-\delta_{p}(s)}^{s}\left(\left(x_{p}^{R}\right)^{\Delta}(u)-\left(x_{p}^{* R}\right)^{\Delta}(u)\right) \Delta u\right. \\
& +\sum_{q=1}^{n}\left(a _ { p q } ^ { R } ( s ) \left(f_{q}^{R}\left(x_{q}^{R}\left(s-\tau_{p q}(s)\right)\right)\right.\right. \\
& \left.-f_{q}^{R}\left(x_{q}^{* R}\left(s-\tau_{p q}(s)\right)\right)\right)
\end{aligned}
$$




$$
\begin{aligned}
& -a_{p q}^{I}(s)\left(f_{q}^{I}\left(x_{q}^{I}\left(s-\tau_{p q}(s)\right)\right)-f_{q}^{I}\left(x_{q}^{* I}\left(s-\tau_{p q}(s)\right)\right)\right) \\
& -a_{p q}^{J}(s)\left(f_{q}^{J}\left(x_{q}^{J}\left(s-\tau_{p q}(s)\right)\right)-f_{q}^{J}\left(x_{q}^{* J}\left(s-\tau_{p q}(s)\right)\right)\right) \\
& -a_{p q}^{K}(s)\left(f_{q}^{K}\left(x_{q}^{K}\left(s-\tau_{p q}(s)\right)\right)\right. \\
& \left.\left.-f_{q}^{K}\left(x_{q}^{* K}\left(s-\tau_{p q}(s)\right)\right)\right)\right) \\
& +\sum_{q=1}^{n}\left(b _ { p q } ^ { R } ( s ) \left(g_{q}^{R}\left(\left(x_{q}^{R}\right)^{\Delta}\left(s-\eta_{p q}(s)\right)\right)\right.\right. \\
& \left.-g_{q}^{R}\left(\left(x_{q}^{* R}\right)^{\Delta}\left(s-\eta_{p q}(s)\right)\right)\right) \\
& -b_{p q}^{I}(s)\left(g_{q}^{I}\left(\left(x_{q}^{I}\right)^{\Delta}\left(s-\eta_{p q}(s)\right)\right)\right. \\
& \left.-g_{q}^{I}\left(\left(x_{q}^{* I}\right)^{\Delta}\left(s-\eta_{p q}(s)\right)\right)\right) \\
& -b_{p q}^{J}(s)\left(g_{q}^{J}\left(\left(x_{q}^{J}\right)^{\Delta}\left(s-\eta_{p q}(s)\right)\right)\right. \\
& \left.-g_{q}^{J}\left(\left(x_{q}^{* J}\right)^{\Delta}\left(s-\eta_{p q}(s)\right)\right)\right) \\
& -b_{p q}^{K}(s)\left(g_{q}^{K}\left(\left(x_{q}^{K}\right)^{\Delta}\left(s-\eta_{p q}(s)\right)\right)\right. \\
& \left.\left.\left.-g_{q}^{K}\left(\left(x_{q}^{* K}\right)^{\Delta}\left(s-\eta_{p q}(s)\right)\right)\right)\right)\right) \Delta s \mid \\
& \leq e_{-c_{p}}\left(t_{1}, 0\right)\left(\|\phi\|_{0}+\epsilon\right) \\
& +c M e_{\ominus \lambda}\left(t_{1}, 0\right)\left(\|\phi\|_{0}+\epsilon\right) \\
& \cdot \int_{0}^{t_{1}} e_{-c_{p} \oplus \lambda}\left(t_{1}, \sigma(s)\right)\left(c_{p}^{+} \delta_{p}^{+} \times e_{\lambda}\left(\sigma(s), s-\delta_{p}(s)\right)\right. \\
& +A_{p}^{R} e_{\lambda}\left(\sigma(s), s-\tau_{p q}(s)\right) \\
& \left.+B_{p}^{R} e_{\lambda}\left(\sigma(s), s-\eta_{p q}(s)\right)\right) \Delta s \\
& \leq c M e_{\ominus \lambda}\left(t_{1}, 0\right)\left(\|\phi\|_{0}+\epsilon\right) \\
& \text {. }\left\{\frac{e_{-c_{p} \oplus \lambda}\left(t_{1}, 0\right)}{c M}+\int_{0}^{t_{1}} e_{-c_{p} \oplus \lambda}\left(t_{1}, \sigma(s)\right)\right. \\
& \cdot \exp \left(\lambda \sup _{s \in \mathbb{\mathbb { N }}} \mu(s)\right) \\
& \times\left(c_{p}^{+} \delta_{p}^{+} \exp \left(\lambda \delta_{p}^{+}\right)+A_{p}^{R} \exp \left(\lambda \tau_{p q}^{+}\right)\right. \\
& \left.\left.+B_{p}^{R} \exp \left(\lambda \eta_{p q}^{+}\right)\right) \Delta s\right\} \\
& <c M e_{\ominus \lambda}\left(t_{1}, 0\right)\left(\|\phi\|_{0}+\epsilon\right) \\
& \cdot\left\{\frac{e_{-\left(c_{p}-\lambda\right)}\left(t_{1}, 0\right)}{M}+\frac{1-e_{-\left(c_{p}-\lambda\right)}\left(t_{1}, 0\right)}{c_{p}^{-}-\lambda}\right. \\
& \cdot \exp \left(\lambda \sup _{s \in \mathbb{T}} \mu(s)\right) \\
& \times\left(c_{p}^{+} \delta_{p}^{+} \exp \left(\lambda \delta_{p}^{+}\right)+A_{p}^{R} \exp \left(\lambda \tau_{p q}^{+}\right)\right. \\
& \left.\left.+B_{p}^{R} \exp \left(\lambda \eta_{p q}^{+}\right)\right)\right\}
\end{aligned}
$$$$
=c M e_{\ominus \lambda}\left(t_{1}, 0\right)\left(\|\phi\|_{0}+\epsilon\right)
$$

$$
\begin{aligned}
& \cdot\left\{\left[\frac{1}{M}-\frac{\exp \left(\lambda \sup _{s \in \mathbb{T}} \mu(s)\right)}{c_{p}^{-}-\lambda}\right.\right. \\
& \cdot\left(c_{p}^{+} \delta_{p}^{+} \exp \left(\lambda \delta_{p}^{+}\right)+A_{p}^{R} \exp \left(\lambda \tau_{p q}^{+}\right)\right. \\
& \left.\left.+B_{p}^{R} \exp \left(\lambda \eta_{p q}^{+}\right)\right)\right] e_{-\left(c_{p}-\lambda\right)}\left(t_{1}, 0\right) \\
& +\frac{\exp \left(\lambda \sup _{s \in \mathbb{T}} \mu(s)\right)}{c_{p}^{-}-\lambda} \\
& \cdot\left(c_{p}^{+} \delta_{p}^{+} \exp \left(\lambda \delta_{p}^{+}\right)+A_{p}^{R} \exp \left(\lambda \tau_{p q}^{+}\right)\right. \\
& \left.\left.+B_{p}^{R} \exp \left(\lambda \eta_{p q}^{+}\right)\right)\right\} \\
& <c M e_{\ominus \lambda}\left(t_{1}, 0\right)\left(\|\phi\|_{0}+\epsilon\right), \quad p \in \Lambda .
\end{aligned}
$$

Similarly, we can get

$$
\begin{aligned}
\left|\left(x-x^{*}\right)_{p}^{l}\left(t_{1}\right)\right|<c M e_{\ominus \lambda}\left(t_{1}, 0\right)\left(\|\phi\|_{0}+\epsilon\right), & \\
& p \in \Lambda, l=I, J, K .
\end{aligned}
$$

On the other hand, we have

$$
\begin{aligned}
& \left|\left(\left(x-x^{*}\right)_{p}^{R}\right)^{\Delta}\left(t_{1}\right)\right| \leq-c_{p}^{+} e_{-c_{p}}\left(t_{1}, 0\right)\left(\|\phi\|_{0}+\epsilon\right) \\
& +c M e_{\ominus \lambda}\left(t_{1}, 0\right)\left(\|\phi\|_{0}+\epsilon\right)\left(c _ { p } ^ { + } \delta _ { p } ^ { + } e _ { \lambda } \left(t_{1}, t_{1}\right.\right. \\
& \left.-\delta_{p}\left(t_{1}\right)\right)+A_{p}^{R} e_{\lambda}\left(t_{1}, t_{1}-\tau_{p q}\left(t_{1}\right)\right)+B_{p}^{R} e_{\lambda}\left(t_{1}, t_{1}\right. \\
& \left.\left.-\eta_{p_{1} q}\left(t_{1}\right)\right)\right)+c_{p}^{+} c M e_{\ominus \lambda}\left(t_{1}, 0\right)\left(\|\phi\|_{0}+\epsilon\right) \\
& \times\left(c_{p}^{+} \delta_{p}^{+} e_{\lambda}\left(\sigma(s), s-\delta_{p}(s)\right)+A_{p}^{R} e_{\lambda}(\sigma(s), s\right. \\
& \left.\left.-\tau_{p q}(s)\right)+B_{p}^{R} e_{\lambda}\left(\sigma(s), s-\eta_{p q}(s)\right)\right) \Delta s \\
& <c M e_{\ominus \lambda}\left(t_{1}, 0\right)\left(\|\phi\|_{0}+\epsilon\right)\left\{\left[\frac{1}{M}\right.\right. \\
& -\frac{\exp \left(\lambda \sup _{s \in \mathbb{T}} \mu(s)\right)}{c_{p}^{-}-\lambda}\left(c_{p}^{+} \delta_{p}^{+} \exp \left(\lambda \delta_{p}^{+}\right)\right. \\
& \left.\left.+A_{p}^{R} \exp \left(\lambda \tau_{p q}^{+}\right)+B_{p}^{R} \exp \left(\lambda \eta_{p_{1} q}^{+}\right)\right)\right] c_{p}^{+} e_{-\left(c_{p}-\lambda\right)}\left(t_{1},\right. \\
& 0)+\left(1+\frac{c_{p}^{+} \exp \left(\lambda \sup _{s \in \mathbb{T}} \mu(s)\right)}{c_{p}^{-}-\lambda}\right) \times\left(c_{p}^{+} \delta_{p}^{+}\right. \\
& \left.\left.\cdot \exp \left(\lambda \delta_{p}^{+}\right)+A_{p}^{R} \exp \left(\lambda \tau_{p q}^{+}\right)+B_{p}^{R} \exp \left(\lambda \eta_{p q}^{+}\right)\right)\right\} \\
& <c M e_{\ominus \lambda}\left(t_{1}, 0\right)\left(\|\phi\|_{0}+\epsilon\right), \quad p \in \Lambda \text {. }
\end{aligned}
$$


Similarly, we have

$$
\begin{array}{r}
\left|\left(\left(x-x^{*}\right)_{p}^{l}\right)^{\Delta}\left(t_{1}\right)\right|<c M e_{\ominus \lambda}\left(t_{1}, 0\right)\left(\|\phi\|_{0}+\epsilon\right), \\
p \in \Lambda, l=I, J, K .
\end{array}
$$

It follows from (52) to (55) that

$$
\left\|Z\left(t_{1}\right)\right\|<c M e_{\ominus \lambda}\left(t_{1}, 0\right)\left(\|\phi\|_{0}+\epsilon\right)
$$

which contradicts the first equation of (49). Therefore, (48) holds. Let $\epsilon \rightarrow 0^{+}$leads to

$$
\|Z(t)\| \leq M e_{\ominus \lambda}(t, 0)\|\phi\|_{0}, \quad \forall t \in(0,+\infty)_{\mathbb{T}} .
$$

Hence, the pseudo almost periodic solution of system (17) is globally exponentially stable. The proof is complete.

Remark 13. From Theorems 11 and 12, we can find that the time delays in the leakage term are harmful for the existence and stability of almost periodic solutions of quaternionvalued system (1). Therefore, the time delays in the leakage term cannot be ignored.

Remark 14. In view of Theorems 11 and 12, we can also find that the neutral terms have an influence on the existence and stability of the almost periodic solution. Therefore, they cannot be ignored too.

Remark 15. According to Theorems 11 and 12, it is clear that if the coefficients of leakage terms of (1) are positive regressive, then the continuous-time network and its corresponding discrete-time network have the same dynamics for the pseudo almost periodicity.

\section{An Example}

In this section, we give an example to illustrate the feasibility and effectiveness of our results obtained in Section 3.

Example 1. Let $n=2$. Consider the following quaternionvalued neural networks with time delays on almost periodic time scale $\mathbb{T}$ :

$$
\begin{aligned}
x_{p}^{\Delta}(t)= & -c_{p}(t) x_{p}\left(t-\delta_{p}(t)\right) \\
& +\sum_{q=1}^{n} a_{p q}(t) f_{q}\left(x_{q}\left(t-\tau_{p q}(t)\right)\right) \\
& +\sum_{q=1}^{n} b_{p q}(t) g_{q}\left(x_{q}^{\Delta}\left(t-\eta_{p q}(t)\right)\right)+u_{p}(t),
\end{aligned}
$$

where $p=1,2, t \in \mathbb{T}$ and the coefficients are as follows:

$$
\begin{aligned}
& c_{1}(t)=0.4+0.1|\sin \sqrt{2} t|, \\
& c_{2}(t)=0.5-0.1|\sin t|, \\
& f_{1}\left(x_{1}\right)=f_{2}\left(x_{1}\right) \\
& =\frac{1}{15} \sin x_{1}^{R}+i \frac{1}{15}\left|\cos x_{1}^{I}-1\right|+j \frac{1}{30} \sin ^{2} x_{1}^{J} \\
& +k \frac{1}{15} \tanh x_{1}^{K}, \\
& g_{1}\left(x_{1}\right)=g_{2}\left(x_{1}\right) \\
& =\frac{1}{32}\left(\left|x_{1}^{R}+1\right|+\left|x_{1}^{R}\right|-1\right)+i \frac{1}{32} \sin ^{2} x_{1}^{I} \\
& +j \frac{1}{16}\left|x_{2}^{J}\right|+k \frac{1}{32} \sin ^{2} x_{2}^{K}, \\
& a_{11}(t)=a_{12}(t) \\
& =0.2|\cos (\sqrt{2} t)|+i 0.1|\sin (\sqrt{3} t)| \\
& +j 0.24|\cos t|+k 0.26|\sin t| \text {, } \\
& a_{21}(t)=a_{22}(t) \\
& =0.28|\sin (\sqrt{2} t)|+i 0.32|\cos t| \\
& +j 0.25|\cos (\sqrt{2} t)|+k 0.22|\cos t|, \\
& b_{11}(t)=b_{12}(t) \\
& =0.16|\cos t|+i 0.2|\cos (\sqrt{3} t)| \\
& +j 0.15|\sin (\sqrt{3} t)|+k 0.25|\cos (\sqrt{2} t)| \text {, } \\
& b_{21}(t)=b_{22}(t) \\
& =0.2|\sin (\sqrt{3} t)|+i 0.18|\sin t|+j 0.3|\cos t| \\
& +k 0.26|\sin (\sqrt{2} t)| \text {, } \\
& u_{1}(t)=u_{2}(t) \\
& =0.1 \cos (\sqrt{2} t)+i 0.15 \sin t \\
& +j 0.09 \sin (\sqrt{2} t)+k 0.12 \cos (\sqrt{2} t), \\
& \delta_{1}(t)=0.01|\sin (2 \pi t)|, \\
& \delta_{2}(t)=0.02 \sin ^{2}(\pi t), \\
& \tau_{p q}(t)=0.2\left|\cos \left(\pi t+\frac{\pi}{2}\right)\right|, \\
& \eta_{p q}(t)=0.3\left|\cos \left(\pi t+\frac{3 \pi}{2}\right)\right| \text {, }
\end{aligned}
$$



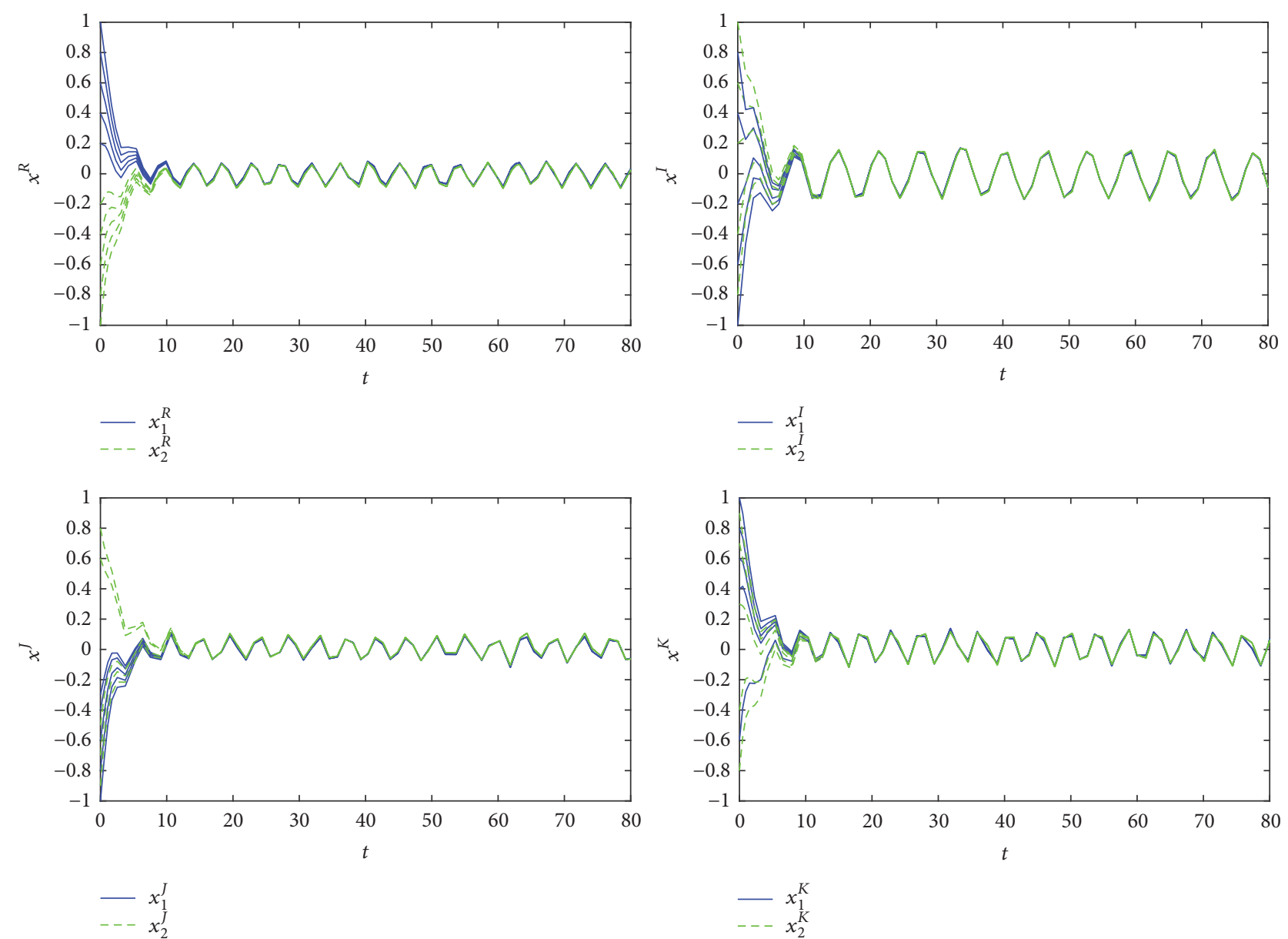

FIGURE 1: Transient states of four parts with continuous time $t$ of (58) in Example 1.

Obviously, $\left(H_{1}\right)$ holds. By calculating, we have

$$
\begin{aligned}
& c_{1}^{-}=0.3, \\
& c_{2}^{-}=0.4, \\
& c_{1}^{+}=0.4, \\
& c_{2}^{+}=0.5, \\
& \alpha_{1}^{l}=\alpha_{2}^{l}=\frac{1}{15}, \\
& \beta_{1}^{l}=\beta_{2}^{l}=\frac{1}{16},
\end{aligned}
$$

$l \in E$,

$$
\begin{aligned}
& a_{11}^{R^{+}}=a_{12}^{R^{+}}=0.2, \\
& a_{11}^{I^{+}}=a_{12}^{I^{+}}=0.1, \\
& a_{11}^{J^{+}}=a_{12}^{J^{+}}=0.24, \\
& a_{11}^{K^{+}}=a_{12}^{K^{+}}=0.26,
\end{aligned}
$$

$$
\begin{aligned}
& a_{21}^{R^{+}}=a_{22}^{R^{+}}=0.28, \\
& a_{21}^{I^{+}}=a_{22}^{I^{+}}=0.32, \\
& a_{21}^{J^{+}}=a_{22}^{J^{+}}=0.25, \\
& a_{21}^{K^{+}}=a_{22}^{K^{+}}=0.22, \\
& b_{11}^{R^{+}}=b_{12}^{R^{+}}=0.16, \\
& b_{11}^{I^{+}}=b_{12}^{I^{+}}=0.2, \\
& b_{11}^{J^{+}}=b_{12}^{J^{+}}=0.15, \\
& b_{11}^{K^{+}}=b_{12}^{K^{+}}=0.25, \\
& b_{21}^{R^{+}}=b_{22}^{R^{+}}=0.2, \\
& b_{21}^{I^{+}}=b_{22}^{I^{+}}=0.18, \\
& b_{21}^{J^{+}}=b_{22}^{J^{+}}=0.3, \\
& b_{21}^{K^{+}}=b_{22}^{K^{+}}=0.26, \\
& u_{1}^{R^{+}}=u_{2}^{R^{+}}=0.1,
\end{aligned}
$$



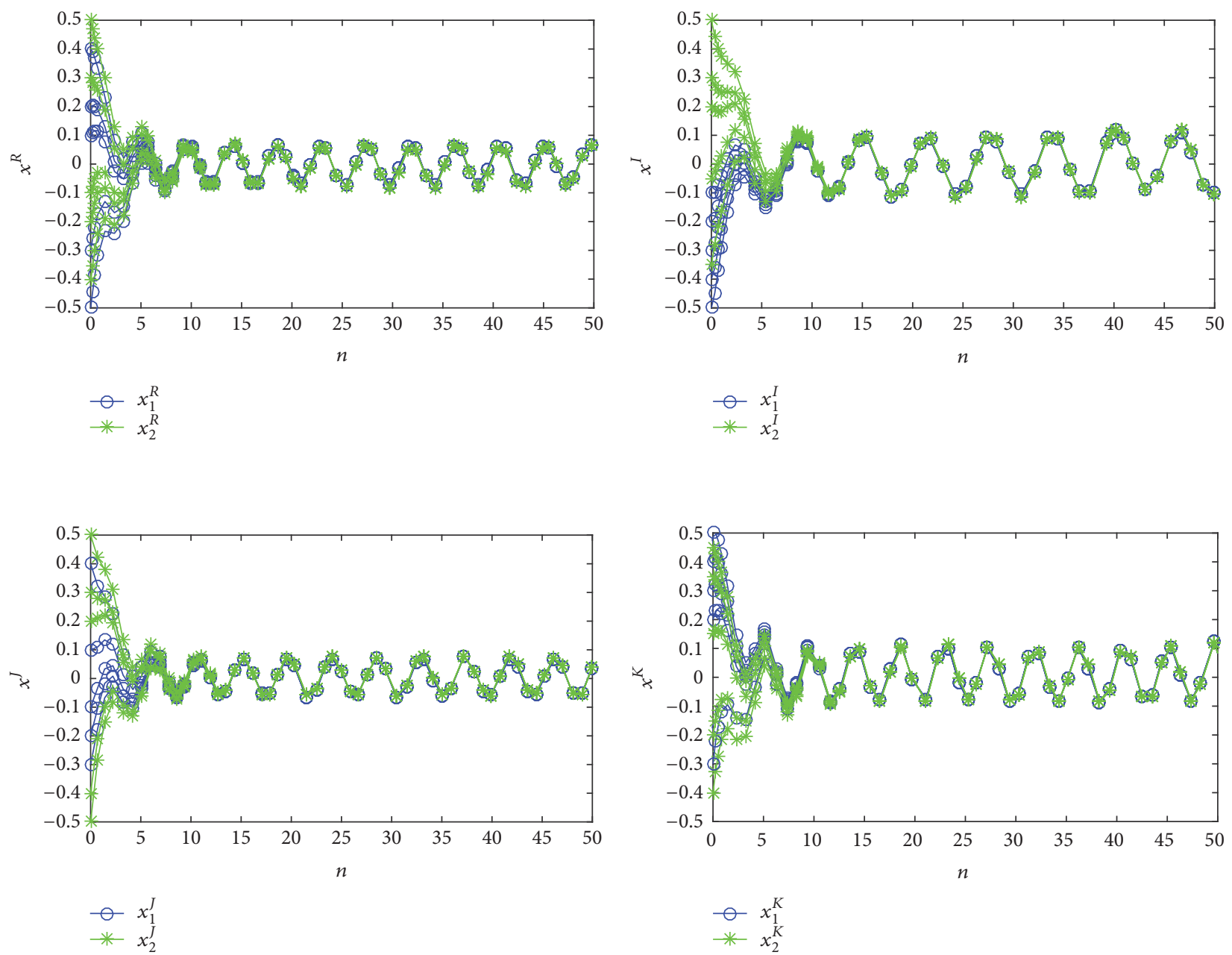

FIGURE 2: Transient states of four parts with discrete time $t$ of (58) in Example 1.

$$
\begin{aligned}
& u_{1}^{I^{+}}=u_{2}^{I^{+}}=0.15, \\
& u_{1}^{J^{+}}=u_{2}^{J^{+}}=0.09, \\
& u_{1}^{K^{+}}=u_{2}^{K^{+}}=0.12, \\
& \delta_{1}^{+}=0.01, \\
& \delta_{2}^{+}=0.02, \\
& \tau_{p q}^{+}=0.2, \\
& \eta_{p q}^{+}=0.3,
\end{aligned}
$$

$$
\begin{aligned}
& \max _{1 \leq p \leq 2}\left\{\max _{l \in E}\left\{\frac{\Gamma_{p}^{l} \kappa+u_{p}^{l^{+}}}{c_{p}^{-}},\left(1+\frac{c_{p}^{+}}{c_{p}^{-}}\right)\left(\Gamma_{p}^{l} \kappa+u_{p}^{l^{+}}\right)\right\}\right\} \\
& =1.8713<\kappa=2, \\
& \max _{1 \leq p \leq 2}\left\{\max _{l \in E}\left\{\frac{\Gamma_{p}^{l}}{c_{p}^{-}},\left(1+\frac{c_{p}^{+}}{c_{p}^{-}}\right) \Gamma_{p}^{l}\right\}\right\}=0.6857=\rho<1 .
\end{aligned}
$$

Therefore, whether $\mathbb{T}=\mathbb{R}$ or $\mathbb{T}=\mathbb{Z}$, all the conditions of Theorems 11 and 12 are satisfied; hence, we know that system (58) has a unique pseudo almost periodic solution, which is globally exponentially stable. This is, the continuous-time neural network and its discrete-time analogue have the same

$$
p, q=1,2
$$

It is easy to see that the following conditions hold. Take $\kappa=2$; then, we have

$$
\begin{aligned}
& \Gamma_{1}^{R}=\Gamma_{1}^{I}=\Gamma_{1}^{J}=\Gamma_{1}^{K}=0.2057, \\
& \Gamma_{2}^{R}=\Gamma_{2}^{I}=\Gamma_{2}^{J}=\Gamma_{2}^{K}=0.2702,
\end{aligned}
$$
dynamical behaviors for the pseudo almost periodicity (see Figures 1 and 2).

\section{Conclusion}

In this paper, we have proposed a class of quaternion-valued neural networks of neutral type with delays in the leakage term on time scales. Based on the exponential dichotomy of linear dynamic equations on time scales, Banach's fixed 
point theorem and the theory of calculus on time scales, we obtain some sufficient conditions on the existence and global exponential stability of pseudo almost periodic solutions for the quaternion-valued neural networks. An example has been given to demonstrate the effectiveness of our results. To the best of our knowledge, this is the first time to study the pseudo almost periodic solutions for quaternion-valued neural networks on time scales. Our methods used in this paper can be applied to study other types of quaternionvalued systems on times scales.

\section{Conflicts of Interest}

The authors declare that they have no conflicts of interest.

\section{Acknowledgments}

This work is supported by the National Natural Sciences Foundation of People's Republic of China under Grant 11361072.

\section{References}

[1] W. R. Hamilton, "Lectures on Quaternions," in $s$ and Smith, Hodges, Dublin, 1853

[2] R. Mukundan, "Quaternions: From Classical Mechanics to Computer Graphics, and Beyond," in Proceedings of the 7th Asian Technology Conference in Mathematics, pp. 95-105, 2002.

[3] J. B. Kuipers, Quaternions and Rotation Sequences, Princeton University Press, Princeton, NJ, USA, 1999.

[4] S. G. Hoggar, Mathematics for Computer Graphics, Cambridge University Press, Cambridge, UK, 1992.

[5] X. Chen, Z. Li, Q. Song, J. Hu, and Y. Tan, "Robust stability analysis of quaternion-valued neural networks with time delays and parameter uncertainties," Neural Networks, vol. 91, pp. 55-65, 2017.

[6] H. Shu, Q. Song, Y. Liu, Z. Zhao, and F. E. Alsaadi, "Global $\mu$-stability of quaternion-valued neural networks with nondifferentiable time-varying delays," Neurocomputing, vol. 247, pp. 202-212, 2017.

[7] L. Saad Saoud, R. Ghorbani, and F. Rahmoune, "Cognitive Quaternion Valued Neural Network and some applications," Neurocomputing, vol. 221, pp. 85-93, 2017.

[8] J. Hu, C. Zeng, and J. Tan, "Boundedness and periodicity for linear threshold discrete-time quaternion-valued neural network with time-delays," Neurocomputing, vol. 267, pp. 417-425, 2017.

[9] L. Xiaodong, L. Aijun, Y. Changjun, and S. Fulin, "Widely Linear Quaternion Unscented Kalman Filter for QuaternionValued Feedforward Neural Network," IEEE Signal Processing Letters, pp. 1-1.

[10] P. Arena, R. Caponetto, L. Fortuna, G. Muscato, and M. G. Xibilia, "Quaternionic multilayer perceptrons for chaotic time series prediction," IEICE Transactions on Fundamentals of Electronics, Communications and Computer Sciences, vol. E79A, no. 10, pp. 1682-1687, 1996.

[11] P. Arena, L. Fortuna, G. Muscato, and M. G. Xibilia, "Multilayer perceptrons to approximate quaternion valued functions," $\mathrm{Neu}$ ral Networks, vol. 10, no. 2, pp. 335-342, 1997.

[12] C. Jahanchahi, C. Cheong Took, and D. P. Mandic, "On HR calculus, quaternion valued stochastic gradient, and adaptive three dimensional wind forecasting," in Proceedings of the 2010 6th IEEE World Congress on Computational Intelligence, WCCI 2010 - 2010 International Joint Conference on Neural Networks, IJCNN 2010, Spain, July 2010.

[13] B. C. Ujang, C. C. Took, and D. P. D. Mandic, "Quaternionvalued nonlinear adaptive filtering," IEEE Transactions on Neural Networks and Learning Systems, vol. 22, no. 8, pp. 11931206, 2011.

[14] H. Kusamichi, T. Isokawa, N. Matsui, Y. Ogawa, and K. Maeda, "A new scheme color night vision by quaternion neural network," in Proceedings of the in Proceedings of the 2nd International Conference on Autonomous Robots and Agents, pp. 101106, 2004.

[15] L. Luo, H. Feng, and L. Ding, "Color image compression based on quaternion neural network principal component analysis," in Proceedings of the 2010 International Conference on Multimedia Technology, ICMT 2010, China, October 2010.

[16] L. Ding and H. Feng, "Quaternion K-L transform and biomimetic pattern recognition approaches for color-face recognition," in Proceedings of the 2009 IEEE International Conference on Intelligent Computing and Intelligent Systems, ICIS 2009, pp. 165-169, China, November 2009.

[17] M. Wang, C. C. Took, and D. P. Mandic, "A class of fast quaternion valued variable stepsize stochastic gradient learning algorithms for vector sensor processes," in Proceedings of the 2011 International Joint Conference on Neural Network, IJCNN 2011, pp. 2783-2786, USA, August 2011.

[18] S. Mohamad and K. Gopalsamy, "Exponential stability of continuous-time and discrete-time cellular neural networks with delays," Applied Mathematics and Computation, vol. 135, no. 1, pp. 17-38, 2003.

[19] Y. Li, "Global exponential stability of BAM neural networks with delays and impulses," Chaos, Solitons \& Fractals, vol. 24, no. 1, pp. 279-285, 2005.

[20] J. Cao and Q. Song, "Stability in Cohen-Grossberg-type bidirectional associative memory neural networks with time-varying delays," Nonlinearity, vol. 19, no. 7, pp. 1601-1617, 2006.

[21] Q. Zhu, J. Cao, and R. Rakkiyappan, "Exponential input-to-state stability of stochastic Cohen-Grossberg neural networks with mixed delays," Nonlinear Dynamics, vol. 79, no. 2, pp. 1085-1098, 2014.

[22] C.-K. Zhang, Y. He, L. Jiang, Q. H. Wu, and M. Wu, "Delaydependent stability criteria for generalized neural networks with two delay components," IEEE Transactions on Neural Networks and Learning Systems, vol. 25, no. 7, pp. 1263-1276, 2014.

[23] R. Rakkiyappan, J. Cao, and G. Velmurugan, "Existence and uniform stability analysis of fractional-order complex-valued neural networks with time delays," IEEE Transactions on Neural Networks and Learning Systems, vol. 26, no. 1, pp. 84-97, 2015.

[24] S. Arik, "An improved robust stability result for uncertain neural networks with multiple time delays," Neural Networks, vol. 54, pp. 1-10, 2014.

[25] Q. K. Song and Z. J. Zhao, "Stability criterion of complex-valued neural networks with both leakage delay and time-varying delays on time scales," Neurocomputing, vol. 171, pp. 179-184, 2016.

[26] P. Jiang, Z. Zeng, and J. Chen, "On the periodic dynamics of memristor-based neural networks with leakage and timevarying delays," Neurocomputing, vol. 219, pp. 163-173, 2017. 
[27] C. Y. Zhang, "Pseudo-almost-periodic solutions of some differential equations," Journal of Mathematical Analysis and Applications, vol. 181, no. 1, pp. 62-76, 1994.

[28] C. Y. Zhang, "Pseudo almost periodic solutions of some differential equations," Journal of Mathematical Analysis and Applications, vol. 192, no. 2, pp. 543-561, 1995.

[29] T. Diagana, "Pseudo almost periodic solutions to some differential equations," Nonlinear Analysis. Theory, Methods \& Applications, vol. 60, no. 7, pp. 1277-1286, 2005.

[30] T. Diagana, C. M. Mahop, and G. M. N’Guérékata, “Pseudoalmost-periodic solutions to some semilinear differential equations," Mathematical and Computer Modelling, vol. 43, no. 1-2, pp. 89-96, 2006.

[31] M. Pinto, "Pseudo-almost periodic solutions of neutral integral and differential equations with applications," Nonlinear Analysis. Theory, Methods \& Applications, vol. 72, no. 12, pp. 43774383, 2010.

[32] B. W. Liu, "Pseudo almost periodic solutions for neutral type CNNs with continuously distributed leakage delays," Neurocomputing, vol. 148, pp. 445-454, 2015.

[33] B. Ammar, F. Cherif, and A. M. Alimi, "Existence and uniqueness of pseudo almost-periodic solutions of recurrent neural networks with time-varying coefficients and mixed delays," IEEE Transactions on Neural Networks and Learning Systems, vol. 23, no. 1, pp. 109-118, 2012.

[34] F. Chérif, "Existence and global exponential stability of pseudo almost periodic solution for SICNNs with mixed delays," Applied Mathematics and Computation, vol. 39, no. 1-2, pp. 235251, 2012.

[35] S. Abbas, "Pseudo almost periodic sequence solutions of discrete time cellular neural networks," Lithuanian Association of Nonlinear Analysts. Nonlinear Analysis: Modelling and Control, vol. 14, no. 3, pp. 283-301, 2009.

[36] Y. Yu, "Exponential Stability of Pseudo Almost Periodic Solutions for Cellular Neural Networks with Multi-Proportional Delays," Neural Processing Letters, vol. 45, no. 1, pp. 141-151, 2017.

[37] A. Zhang, "Pseudo Almost Periodic Solutions for SICNNs with Oscillating Leakage Coefficients and Complex Deviating Arguments," Neural Processing Letters, vol. 45, no. 1, pp. 183-196, 2017.

[38] C. Aouiti, M. S. M'hamdi, and A. Touati, "Pseudo Almost Automorphic Solutions of Recurrent Neural Networks with TimeVarying Coefficients and Mixed Delays," Neural Processing Letters, vol. 45, no. 1, pp. 121-140, 2017.

[39] Y. Li, L. Yang, and B. Li, "Existence and Stability of Pseudo Almost Periodic Solution for Neutral Type High-Order Hopfield Neural Networks with Delays in Leakage Terms on Time Scales," Neural Processing Letters, vol. 44, no. 3, pp. 603-623, 2016.

[40] S. Hilger, "Analysis on measure chains-a unified approach to continuous and discrete calculus," Results in Mathematics, vol. 18, no. 1-2, pp. 18-56, 1990.

[41] M. Bohner and A. Peterson, Dynamic Equations on Time Scales: An Introduction with Applications, Birkhauser, Boston, Mass, USA, 2001.

[42] M. Bohner and A. Peterson, Advances in Dynamic Equations on Time Scales, Birkhäuser, Boston, Mass, USA, 2003.

[43] M. Federson, R. Grau, J. G. Mesquita, and E. Toon, "Boundedness of solutions of measure differential equations and dynamic equations on time scales," Journal of Differential Equations, vol. 263, no. 1, pp. 26-56, 2017.
[44] S.-Y. Zhang, Q.-R. Wang, and Q. Kong, "Asymptotics and oscillation of $n$ th-order nonlinear dynamic equations on time scales," Applied Mathematics and Computation, vol. 275, pp. 324-334, 2016.

[45] H. Jafari, A. Haghbin, S. J. Johnston, and D. Baleanu, "A new algorithm for solving dynamic equations on a time scale," Journal of Computational and Applied Mathematics, vol. 312, pp. 167-173, 2017.

[46] Y. Li and C. Wang, "Almost periodic functions on time scales and applications," Discrete Dynamics in Nature and Society, vol. 2011, Article ID 727068, 20 pages, 2011.

[47] Y. Li and C. Wang, "Uniformly almost periodic functions and almost periodic solutions to dynamic equations on time scales," Abstract and Applied Analysis, vol. 2011, Article ID 341520, 2011.

[48] M. Bohner, V. Sree Hari Rao, and S. Sanyal, "Global stability of complex-valued neural networks on time scales," Differential Equations and Dynamical Systems, vol. 19, no. 1-2, pp. 3-11, 2011.

[49] B. Du, Y. Liu, H. A. Batarfi, and F. E. Alsaadi, "Almost periodic solution for a neutral-type neural networks with distributed leakage delays on time scales," Neurocomputing, vol. 173, pp. 921-929, 2016.

[50] X. Chen and Q. Song, "Global stability of complex-valued neural networks with both leakage time delay and discrete time delay on time scales," Neurocomputing, vol. 121, pp. 254-264, 2013.

[51] Y. Li, X. Chen, and L. Zhao, "Stability and existence of periodic solutions to delayed Cohen-Grossberg BAM neural networks with impulses on time scales," Neurocomputing, vol. 72, no. 7-9, pp. 1621-1630, 2009.

[52] A. Wu and J.-E. Zhang, "Multistability of memristive neural networks with time-varying delays," Complexity, vol. 21, no. 1, pp. 177-186, 2015.

[53] Y. Li and L. Zhao, "Weighted pseudo-almost periodic functions on time scales with applications to cellular neural networks with discrete delays," Mathematical Methods in the Applied Sciences, vol. 40, no. 6, pp. 1905-1921, 2017.

[54] A. Arbi, A. Alsaedi, and J. Cao, "Delta-Differentiable Weighted Pseudo-Almost Automorphicity on Time-Space Scales for a Novel Class of High-Order Competitive Neural Networks with WPAA Coefficients and Mixed Delays," Neural Processing Letters.

[55] Y. Li and C. Wang, "Pseudo almost periodic functions and pseudo almost periodic solutions to dynamic equations on time scales," Advances in Difference Equations, vol. 2012, article no. 77, 2012.

[56] Y. K. Li, X. F. Meng, and L. L. Xiong, "Pseudo almost periodic solutions for neutral type high-order Hopfield neural networks with mixed time-varying delays and leakage delays on time scales," International Journal of Machine Learning and Cybernetics, vol. 8, no. 6, pp. 1915-1927, 2017. 


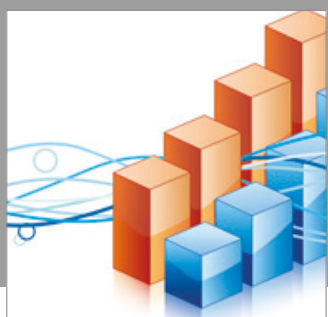

Advances in

Operations Research

vatersals

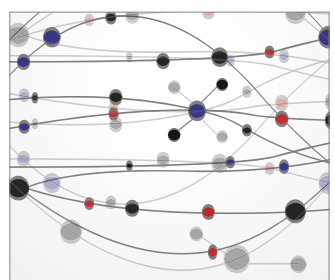

\section{The Scientific} World Journal
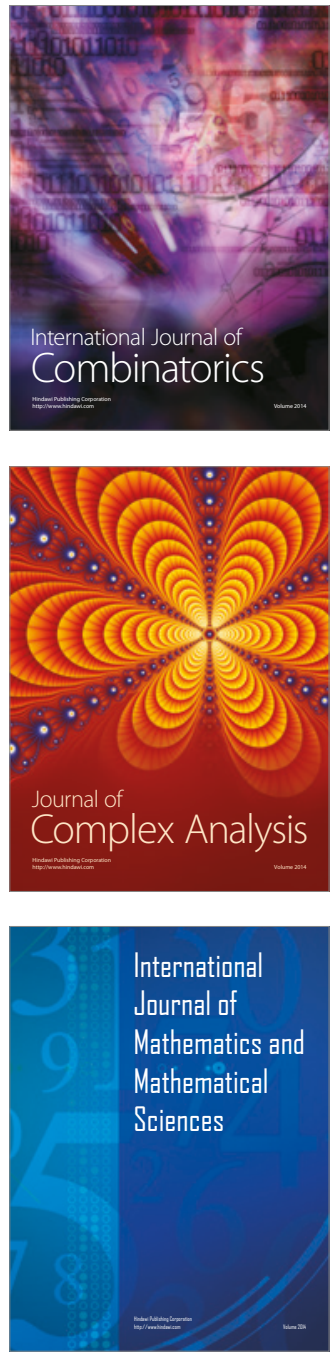
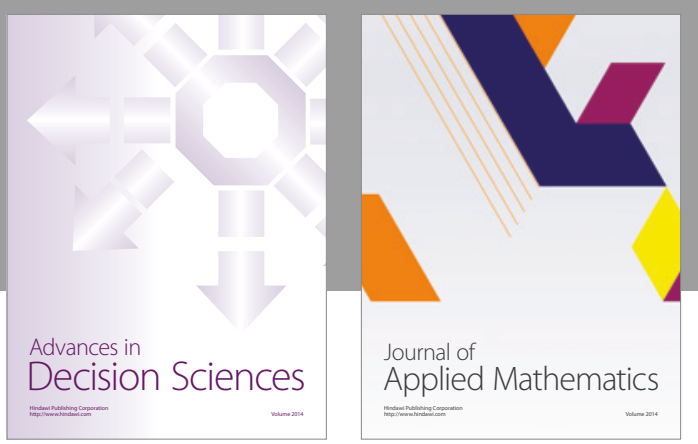

Algebra

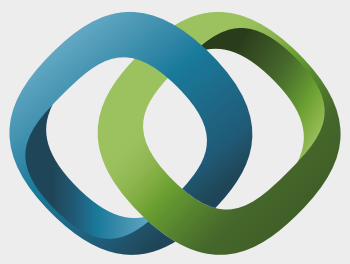

\section{Hindawi}

Submit your manuscripts at

https://www.hindawi.com
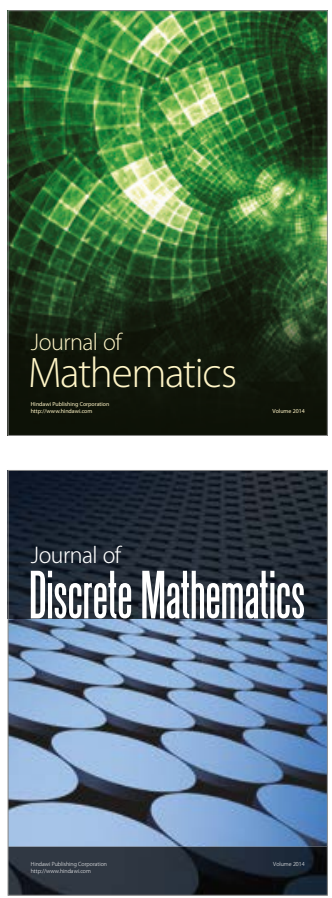

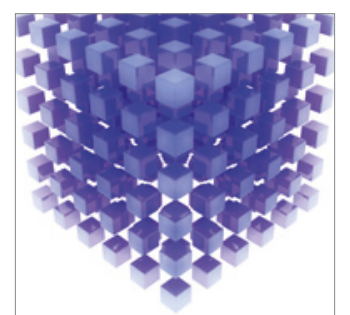

Mathematical Problems in Engineering
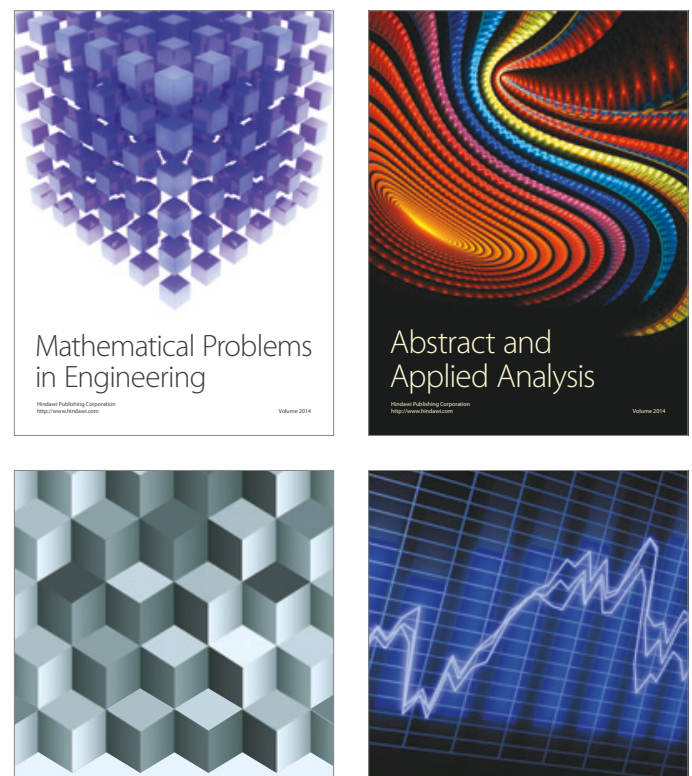

Journal of

Function Spaces

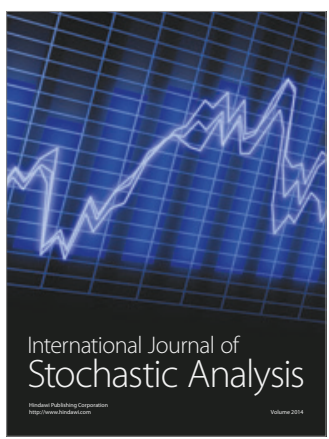

Probability and Statistics
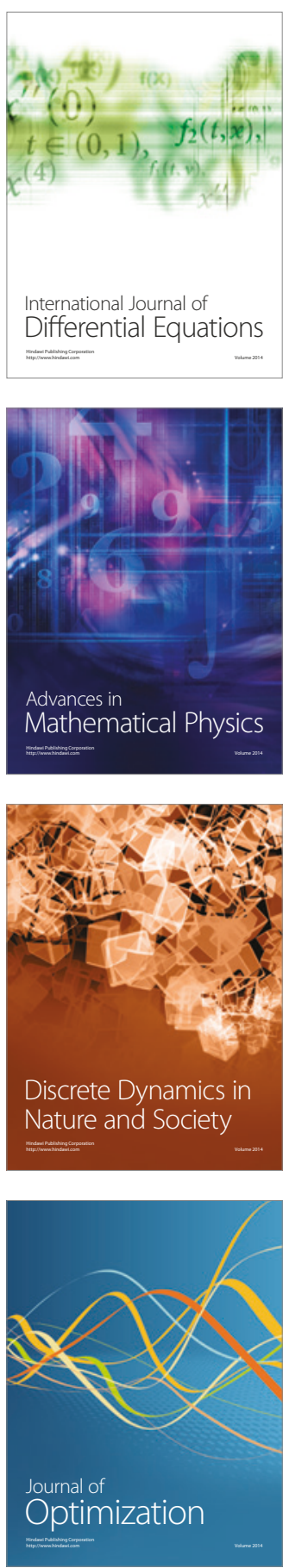